\title{
LOCAL NORMAL FORMS FOR GEODESICALLY EQUIVALENT PSEUDO-RIEMANNIAN METRICS
}

\author{
ALEXEY V. BOLSINOV AND VLADIMIR S. MATVEEV
}

\begin{abstract}
Two pseudo-Riemannian metrics $g$ and $\bar{g}$ are geodesically equivalent if they share the same (unparameterized) geodesics. We give a complete local description of such metrics which solves the natural generalisation of the Beltrami problem for pseudo-Riemannian metrics.
\end{abstract}

\section{INTRODUCTION}

1.1. Definition and history. Two pseudo-Riemannian metrics $g$ and $\bar{g}$ on a manifold $M^{n}$ are called geodesically equivalent if every $g$-geodesic, after an appropriate reparameterisation, is a $\bar{g}$-geodesic. The theory of geodesically equivalent metrics has a long and rich history, the first examples being constructed by Lagrange in 1779 [27. Many important results about geodesically equivalent metrics were obtained by Beltrami [3 5], Levi-Civita [30, Painlevé [40, Lie 31, Liouville 32, Fubini 21], Eisenhart [19, 20], Weyl [48] and Thomas and Veblen [45,47]. Between 1950 and 1990, the theory of geodesically equivalent metrics was one of the main research areas of the Soviet and Japanese differential geometry schools; see the surveys [2,39]. Recently, the theory of geodesically equivalent metrics has had a revival due to new mathematical methods that came from the theory of integrable systems [33] and parabolic Cartan geometry [14,18. These methods have led in the last ten years to the solution of many classical problems, including the Lie problems [13, 38, the Lichnerowicz conjecture [36] and the Weyl-Ehlers problems [24, 37.

In this paper we solve the natural generalization of a problem, first stated by Beltrami, to the case of pseudo-Riemannian metrics, namely:

\section{Beltrami Problem 11 Describe all pairs of geodesically equivalent metrics.}

From context, it is clear that Beltrami actually considered this problem locally and in a neighborhood of almost every point; so do we. It is also clear that Beltrami was considering 2-dimensional Riemannian surfaces; our answer does not have this restriction: the dimension of the manifold and the signatures of the metrics are arbitrary.

Received by the editors January 22, 2013 and, in revised form, October 25, 2013 and February $28,2014$.

2010 Mathematics Subject Classification. Primary 53B30; Secondary 53C50.

The first author was partially supported by Ministry of Education and Science of the Russian Federation (14.B37.21.1935).

The second author was partially supported by DFG (GK 1523) and DAAD (Programm Ostpartnerschaft).

${ }^{1}$ Italian original from [3]: La seconda ... generalizzazione ... del nostro problema, vale a dire: riportare i punti di una superficie sopra un'altra superficie in modo che alle linee geodetiche della prima corrispondano linee geodetiche della seconda. 
Special cases of the Beltrami problem have previously been solved. The twodimensional Riemannian case was solved by Dini [17] in 1869. He showed that two geodesically equivalent Riemannian metrics on a surface in a neighborhood of almost every point are given, in a certain coordinate system, by the following formulas:

(1) $g=(Y(y)-X(x))\left(d x^{2}+d y^{2}\right) \quad$ and $\quad \bar{g}=\left(\frac{1}{X(x)}-\frac{1}{Y(y)}\right)\left(\frac{d x^{2}}{X(x)}+\frac{d y^{2}}{Y(y)}\right)$.

Here $X$ and $Y$ are functions of the indicated variables. For any smooth functions $X, Y$ such that the formulas (11) correspond to Riemannian metrics (i.e., $0<X(x)<$ $Y(y)$ for all $(x, y))$, the metrics $g$ and $\bar{g}$ are geodesically equivalent.

For an arbitrary dimension, the Beltrami problem in the Riemannian case was solved by Levi-Civita 30. We will recall Levi-Civita's 3-dimensional analog of the formulas (1) below in Example 2, page 6723.

The methods of Levi-Civita and Dini cannot be directly generalized to the pseudo-Riemannian case. Levi-Civita and Dini consider the tensor $G$ defined by the condition $g(G \cdot, \cdot)=\bar{g}(\cdot, \cdot)$. Levi-Civita showed that the eigenspaces of this tensor are simultaneously integrable, which implies that (in a neighborhood of almost every point) there exists a local coordinate system,

$$
\left(\bar{x}_{1}, \ldots, \bar{x}_{n}\right)=\left(x_{1}^{1}, \ldots, x_{1}^{m_{1}}, \ldots, x_{k}^{1}, \ldots, x_{k}^{m_{k}}\right),
$$

such that in these coordinates the matrix of $g$ is block diagonal with $k$ blocks of dimension $m_{1}, m_{2}, \ldots, m_{k}$, and the matrix of $G$ is diagonal:

$$
\operatorname{diag}(\underbrace{\rho_{1}, \ldots, \rho_{1}}_{m_{1}}, \ldots, \underbrace{\rho_{k}, \ldots, \rho_{k}}_{m_{k}}) .
$$

In the 2-dimensional Riemannian case, considered by Dini, the existence of such a coordinate system is obvious. In this coordinate system, the partial differential equations on the entries of $g$ and on $\rho_{i}$ expressing the geodesic equivalence condition for $g$ and $\bar{g}=g(G \cdot, \cdot)$ are relatively easy (though they are still coupled) and, after some nontrivial work, can be solved.

The methods of Levi-Civita also work in the pseudo-Riemannian case under the additional assumption that $G$ is diagonalizable. Unfortunately, in the pseudoRiemannian case, the tensor $G$ may have complex eigenvalues and nontrivial Jordan blocks. From the point of view of partial differential equations, the case of many Jordan blocks poses the main difficulty; unlike the case when $G$ is diagonalizable, there is no 'best' coordinate system, and the equations corresponding to the entries of the metrics coming from different blocks are coupled in a very nasty manner.

This difficulty was overcome in 9. In $\$ 1.2$ we recall the main result of 9 and explain that the description of geodesically equivalent metrics $g$ and $\bar{g}$ in a neighborhood of almost every point can be reduced to the case when the tensor $G$ has only one real eigenvalue or two complex conjugate eigenvalues. The biggest part of our paper is devoted to the local description of geodesically equivalent metrics under this assumption.

Special cases of the local description of geodesically equivalent pseudoRiemannian metrics were known before. The 2-dimensional case was described essentially by Darboux [16, $\S \S 593$ and 594]; see also [7,8]. The 3-dimensional case was solved by Petrov [41; it is one of the results for which Petrov obtained the Lenin Prize in 1972, the most important scientific award of the former Soviet Union. 
According to [2], under the additional assumption that the metrics $g$ and $\bar{g}$ have Lorentz signature, the Beltrami problem was solved by Golikov 22 in dimension 4, and by Kruchkovich [26] in all dimensions; unfortunately, we were not able to find and verify these references.

It was generally believed that the Beltrami problem was solved in full generality in 1. Unfortunately, this result of Aminova seems to be wrong. More specifically, in the notation of [1, Theorem 1.1], for $k=1, n=3$ and all $\varepsilon$ 's equal to +1 , the metrics constructed by formulas [1, (1.17), (1.18)] are claimed to be geodesically equivalent, though they are not (the explicit formulas for these metrics can be found in the extended version [10] of the present paper).

1.2. Splitting and gluing construction: Why it is sufficient to assume $G$ has one real eigenvalue or two complex conjugate eigenvalues. Given two metrics $g$ and $\bar{g}$ on the same manifold, instead of considering the (1,1)-tensor $G_{j}^{i}=g^{i k} \bar{g}_{k j}$, we consider the $(1,1)$-tensor $L=L(g, \bar{g})$ defined by

$$
L_{j}^{i}:=\left|\frac{\operatorname{det}(\bar{g})}{\operatorname{det}(g)}\right|^{\frac{1}{n+1}} \bar{g}^{i k} g_{k j},
$$

where $\bar{g}^{i k}$ is the contravariant inverse of $\bar{g}_{i k}$. The tensors $G$ and $L$ are related by

$$
L=|\operatorname{det}(G)|^{\frac{1}{n+1}} G^{-1}, \quad G=\frac{1}{|\operatorname{det}(L)|} L^{-1},
$$

so in particular they have the same structure of Jordan blocks (though their eigenvalues are, in general, different). Since the metric $\bar{g}$ can be uniquely reconstructed from $g$ and $L$, namely,

$$
\bar{g}(\cdot, \cdot)=\frac{1}{|\operatorname{det}(L)|} g\left(L^{-1} \cdot, \cdot\right),
$$

the condition that $\bar{g}$ is geodesically equivalent to $g$ can be written as a system of PDEs on the components of $L$. From the point of view of partial differential equations, the tensor $L$ is more convenient than $G$ : the corresponding system of partial differential equations on $L$ turns out to be linear. In the index-free form, it can be written as the condition (where " $*$ " means $g$-adjoint)

$$
\nabla_{u} L=\frac{1}{2}\left(u \otimes d \operatorname{tr} L+(u \otimes d \operatorname{tr} L)^{*}\right),
$$

which should be fulfilled at every point and for every vector field $u$.

In tensor notation, condition (5) reads

$$
L_{i j, k}=\lambda_{, i} g_{j k}+\lambda_{, j} g_{i k},
$$

where $L_{i j}:=L_{j}^{k} g_{k i}$ and $\lambda:=\frac{1}{2} L_{i}^{i}=\frac{1}{2} \operatorname{tr}(L)$. The tensor $L_{j}^{i}$ defined by (2) is essentially the same as the tensor introduced by Sinjukov (see equations (32), (34) on page 134 of the book [42] and also Theorem 4 on page 135); the equation (6) is also due to him (see also [6, Theorem 2]).

Definition 1.1. We say that a $(1,1)$-tensor $L$ is compatible with $g$ if $L$ is $g$ selfadjoint, nondegenerate at every point and satisfies (5) at any point and for all tangent vectors $u$.

As we explained above, $L$ is compatible with $g$ if and only if $\bar{g}(\cdot, \cdot)=$ $\frac{1}{|\operatorname{det}(L)|} g\left(L^{-1} \cdot, \cdot\right)$ is a pseudo-Riemannian metric geodesically equivalent to $g$. 
The gluing construction, as well as the splitting construction to be presented below, are due to [9]; in the Riemannian case they appeared slightly earlier (see [34, §4], [36, Lemma 2] and [35, §§2.2 and 2.3]).

Consider two pseudo-Riemannian manifolds $\left(M_{1}, h_{1}\right)$ and $\left(M_{2}, h_{2}\right)$. Assume that $L_{1}$ on $M_{1}$ is compatible with $h_{1}$ and $L_{2}$ on $M_{2}$ is compatible with $h_{2}$. Assume in addition that $L_{1}$ and $L_{2}$ have no common eigenvalues in the sense that for any two points $x \in M_{1}, y \in M_{2}$ we have

$$
\text { Spectrum } L_{1}(x) \cap \operatorname{Spectrum} L_{2}(y)=\varnothing .
$$

Then one can naturally and canonically construct a pseudo-Riemannian metric $g$ and a tensor $L$ compatible with $g$ on the direct product $M=M_{1} \times M_{2}$. The new metric $g$ differs from the direct product metric $h_{1}+h_{2}$ on $M_{1} \times M_{2}$ and is given by the following formula involving $L_{1}$ and $L_{2}$. We denote by $\chi_{i}, i=1,2$, the characteristic polynomial of $L_{i}: \chi_{i}=\operatorname{det}\left(t \cdot \mathbf{1}_{i}-L_{i}\right.$ ) (where $\mathbf{1}_{i}$ is the identity operator $\left.\mathbf{1}_{i}: T M_{i} \rightarrow T M_{i}\right)$. We treat the $(1,1)$-tensors $L_{i}$ as linear operators acting on $T M_{i}$. For a polynomial $f(t)=a_{0}+a_{1} t+a_{2} t^{2}+\cdots+a_{m} t^{m}$ and $(1,1)$-tensor $A$, we put $f(A)$ to be the $(1,1)$-tensor

$$
f(A)=a_{0} \cdot \mathbf{1}+a_{1} A+a_{2} A \circ A+\cdots+a_{m} \underbrace{A \circ \cdots \circ A}_{m \text { times }} .
$$

If no eigenvalue of $A$ is a root of $f, f(A)$ is nondegenerate; if $A$ is $g$-selfadjoint, $f(A)$ is $g$-selfadjoint as well.

For two tangent vectors $u=(\underbrace{u_{1}}_{\in T M_{1}}, \underbrace{u_{2}}_{\in T M_{2}}), v=(\underbrace{v_{1}}_{\in T M_{1}}, \underbrace{v_{2}}_{\in T M_{2}}) \in T M$ we put

$$
\begin{aligned}
g(u, v) & =h_{1}\left(\chi_{2}\left(L_{1}\right)\left(u_{1}\right), v_{1}\right)+h_{2}\left(\chi_{1}\left(L_{2}\right)\left(u_{2}\right), v_{2}\right), \\
L(v) & =\left(L_{1}\left(v_{1}\right), L_{2}\left(v_{2}\right)\right) .
\end{aligned}
$$

We see that the $(1,1)$-tensor $L$ is the direct sum of $L_{1}$ and $L_{2}$ in the natural sense.

It might be convenient to understand the formulas (87, 9) in matrix notation: we consider the coordinate system $\left(x_{1}, \ldots, x_{r}, y_{r+1}, \ldots, y_{n}\right)$ on $M$ such that the $x$ 's are coordinates on $M_{1}$ and the $y$ 's are coordinates on $M_{2}$. Then, in this coordinate system, the matrices of $g$ and $L$ have the block diagonal form

$$
g=\left(\begin{array}{cc}
h_{1} \chi_{2}\left(L_{1}\right) & 0 \\
0 & h_{2} \chi_{1}\left(L_{2}\right)
\end{array}\right), \quad L=\left(\begin{array}{cc}
L_{1} & 0 \\
0 & L_{2}
\end{array}\right) .
$$

If (7) is fulfilled, then $g$ is a pseudo-Riemannian metric (i.e., symmetric and nondegenerate) and $L$ is nondegenerate and $g$-selfadjoint.

Theorem 1.2 (Gluing Lemma from [9]). If $L_{1}$ is compatible with $h_{1}$ on $M_{1}, L_{2}$ is compatible with $h_{2}$ on $M_{2}$ and (7) is fulfilled, then $L$ given by (9) is compatible with $g$ given by (8).

Example 1 (Gluing construction and Dini's Theorem). As the manifolds $M_{1}, M_{2}$ we take two intervals $I_{1}$ and $I_{2}$ with coordinates $x$ and $y$ respectively. Next, take the metrics $h_{1}=d x^{2}$ on $I_{1}$ and $h_{2}=-d y^{2}$ on $I_{2}$. Consider the $(1,1)$-tensors $L_{1}=$ $X(x) d x \otimes \frac{\partial}{\partial x}$ on $I_{1}$ and $L_{2}=Y(y) d y \otimes \frac{\partial}{\partial y}$ on $I_{2}$. We assume that $0<X(x)<Y(y)$ for all $x \in I_{1}$ and $y \in I_{2}$. The tensors $L_{1}$ and $L_{2}$ are compatible with $h_{1}$ and $h_{2}$ respectively (which can be checked by direct calculation and which is trivial in 
view of the obvious fact that in dimension 1 all metrics are geodesically equivalent). Then, $\chi_{1}=(t-X(x)), \chi_{2}=(t-Y(y))$, so the formulas (10) read

$$
g=\left(\begin{array}{cc}
Y(y)-X(x) & \\
& Y(y)-X(x)
\end{array}\right), L=\left(\begin{array}{cc}
X(x) & \\
& Y(y)
\end{array}\right) .
$$

Now, combining this with (4), we obtain that this $g$ is geodesically equivalent to the metric

$$
\bar{g}=\frac{Y(y)-X(x)}{X(x) Y(y)}\left(\begin{array}{cc}
\frac{1}{X(x)} & \\
& \frac{1}{Y(y)}
\end{array}\right)=\left(\begin{array}{cc}
\frac{1}{X(x)}-\frac{1}{Y(y)}
\end{array}\right)\left(\begin{array}{cc}
\frac{1}{X(x)} & \\
& \frac{1}{Y(y)}
\end{array}\right) .
$$

Comparing the above formulas with (1), we see that the gluing construction applied to two intervals proves Dini's local description of geodesically equivalent metrics in one direction.

One can iterate this construction: having three pseudo-Riemannian manifolds $\left(M_{1}, h_{1}\right),\left(M_{2}, h_{2}\right),\left(M_{3}, h_{3}\right)$ carrying $h_{i}$-compatible $(1,1)$-tensors $L_{i}$ with pairwise disjoint spectra (see (77)), one can glue $M_{1}$ and $M_{2}$ and then glue the result with $M_{3}$. It is easily seen that the gluing construction is associative and commutative, i.e.

$$
\begin{aligned}
& \left(\left(M_{1}, h_{1}, L_{1}\right) \stackrel{\text { glue }}{+}\left(M_{2}, h_{2}, L_{2}\right)\right) \stackrel{\text { glue }}{+}\left(M_{3}, h_{3}, L_{3}\right) \\
& \quad=\left(M_{1}, h_{1}, L_{1}\right) \stackrel{\text { glue }}{+}\left(\left(M_{2}, h_{2}, L_{2}\right) \stackrel{\text { glue }}{+}\left(M_{3}, h_{3}, L_{3}\right)\right)
\end{aligned}
$$

and

$$
\left(M_{1}, h_{1}, L_{1}\right) \stackrel{\text { glue }}{+}\left(M_{2}, h_{2}, L_{2}\right) \stackrel{\text { iso }}{=}\left(M_{2}, h_{2}, L_{2}\right) \stackrel{\text { glue }}{+}\left(M_{1}, h_{1}, L_{1}\right),
$$

where $\stackrel{\text { iso" }}{=}$ means the existence of a diffeomorphism that preserves the metric and $L$. Actually, this diffeomorphism is given by the natural formula

$$
M_{1} \times M_{2} \ni(\underbrace{x}_{\in M_{1}}, \underbrace{y}_{\in M_{2}}) \mapsto(\underbrace{y}_{\in M_{2}}, \underbrace{x}_{\in M_{1}}) \in M_{2} \times M_{1} .
$$

In the case when we "glue" $k$ manifolds $\left(M_{i}, h_{i}\right)(i=1, \ldots, k)$ such that each manifold is equipped with $h_{i}$-compatible $L_{i}$, we obtain a metric $g$ on $M=M_{1} \times$ $\cdots \times M_{k}$ and $g$-compatible $L$ on $M$ such that in the matrix notation in the natural coordinate system they have the form

$$
g=\left(\begin{array}{llll}
h_{1} \chi_{2}\left(L_{1}\right) \cdots \chi_{k}\left(L_{1}\right) & & & \\
& h_{2} \chi_{1}\left(L_{2}\right) \chi_{3}\left(L_{2}\right) \cdots \chi_{k}\left(L_{2}\right) & & \\
& & \ddots & \\
& & h_{k} \chi_{1}\left(L_{k}\right) \cdots \chi_{k-1}\left(L_{k}\right)
\end{array}\right)
$$

and

$$
L=\left(\begin{array}{cccc}
L_{1} & & & \\
& L_{2} & & \\
& & \ddots & \\
& & & L_{k}
\end{array}\right)
$$

Example 2 (Gluing construction and Levi-Civita's Theorem in dim 3). We now take three intervals $I_{1}, I_{2}, I_{3}$ with the coordinates $x$, resp. $y, z$; the metrics $h_{1}=$ $d x^{2}, h_{2}=-d y^{2}, h_{3}=d z^{3} ;$ and the $h_{i}$-compatible $(1,1)$-tensors $L_{1}=X(x) d x \otimes \frac{\partial}{\partial x}$, 
$L_{2}=Y(y) d y \otimes \frac{\partial}{\partial y}$ and $L_{3}=Z(z) d z \otimes \frac{\partial}{\partial z}$. We again assume that the spectra of $L_{i}$ are pairwise disjoint at every point and

$$
0<X(x)<Y(y)<Z(z) \quad \forall x \in I_{1}, \forall y \in I_{2}, \forall z \in I_{3} .
$$

Applying the gluing construction twice, we obtain the metric $g$ and the $(1,1)$-tensor $L$ on $M^{3}=I_{1} \times I_{2} \times I_{3}$ that are compatible and given in the natural coordinate system by:

$$
g=\left(\begin{array}{r}
(Y(y)-X(x))(Z(z)-X(x)) \\
(Y(y)-X(x))(Z(z)-Y(y)) \\
(Z(z)-Y(y))(Z(z)-X(x))
\end{array}\right)
$$

and

$$
L=\left(\begin{array}{lll}
X(x) & & \\
& Y(y) & \\
& & Z(z)
\end{array}\right) .
$$

Combining this with (4), we obtain a special case of Levi-Civita's local description of geodesically equivalent metrics in dimension 3 (where the tensor $G$ has three different eigenvalues).

The splitting construction is the inverse operation. We will describe its local version only, since it is sufficient for our goals.

Suppose $g$ is a pseudo-Riemannian metric on $M^{n}$ and $L$ is compatible with $g$. We consider an arbitrary point $p \in M$.

We take a point $p$ of the manifold such that in the neighborhood $U(p)$ of this point the eigenvalues of $L$ do not bifurcate (i.e., the number of different eigenvalues is constant in the neighborhood). Then, the eigenvalues are smooth possibly complexvalued functions. We denote them by

$$
\lambda_{1}, \bar{\lambda}_{1}, \ldots, \lambda_{r}, \bar{\lambda}_{r}: U(p) \rightarrow \mathbb{C}, \quad \lambda_{r+1}, \ldots, \lambda_{k}: U(p) \rightarrow \mathbb{R},
$$

where the eigenvalue $\bar{\lambda}_{i}$ is complex conjugate to $\lambda_{i}$ and $\operatorname{Im} \lambda_{i} \neq 0$ for $i \leq r$. We assume that the eigenvalue $\lambda_{i}$ has algebraic multiplicity $m_{i}, 2 m_{1}+\cdots+2 m_{r}+$ $m_{r+1}+\cdots+m_{k}=n$.

Next, let us consider the polynomial functions $\chi_{i}: \mathbb{R} \times U(p) \rightarrow \mathbb{R}$ :

$\chi_{i}=\left(t-\lambda_{i}\right)^{m_{i}}\left(t-\bar{\lambda}_{i}\right)^{m_{i}}$ for $i=1, \ldots, r$ and $\chi_{i}=\left(t-\lambda_{i}\right)^{m_{i}}$ for $i=r+1, \ldots, k$,

and the polynomial function $\hat{\chi}:=\hat{\chi}_{1}+\cdots+\hat{\chi}_{k}$, where $\hat{\chi}_{i}=\frac{\chi}{\chi_{i}}$ and $\chi=\operatorname{det}(t \cdot 1-L)$, is the characteristic polynomial of $L$. It is easy to see that the $(1,1)$-tensor $\hat{\chi}(L)$ is $g$-selfadjoint and nondegenerate. Then we can introduce a new pseudo-Riemannian metric $h$ on $U(p)$ by

$$
h(u, v):=g\left(\hat{\chi}(L)^{-1} u, v\right), \quad u, v \in T_{q} M, \quad q \in U(p) .
$$

Theorem 1.3 (Follows from the Splitting Lemma; see $\S 2.1$ of [9]). In a neighborhood of $p$ there exists a coordinate system

$$
\left(\bar{x}_{1}, \ldots, \bar{x}_{k}\right)=\left(x_{1}^{1}, \ldots, x_{1}^{2 m_{1}}, \cdots, x_{r}^{1}, \ldots, x_{r}^{2 m_{r}}, x_{r+1}^{1}, \ldots, x_{r+1}^{m_{r+1}}, \cdots, x_{k}^{1}, \ldots, x_{k}^{m_{k}}\right)
$$


in which the matrices of $h$ and of $L$ are given by

$$
h=\left(\begin{array}{cccc}
h_{1} & & & \\
& h_{2} & & \\
& & \ddots & \\
& & & h_{k}
\end{array}\right), L=\left(\begin{array}{llll}
L_{1} & & & \\
& L_{2} & & \\
& & \ddots & \\
& & & L_{k}
\end{array}\right) \text {. }
$$

Moreover,

- the entries of the blocks $h_{i}$ and $L_{i}$ depend on the coordinates $\bar{x}_{i}$ only;

- for $i=1, \ldots, r$ the eigenvalues of $L_{i}$ are $\lambda_{i}$ and $\bar{\lambda}_{i}$, and for $i=r+1, \ldots, k$ the only eigenvalue of $L_{i}$ is $\lambda_{i}$;

- $L_{i}$ is compatible with $h_{i}$ for every $i=1, \ldots, k$.

Example 3 (Splitting construction and Dini's Theorem). Consider a 2-dimensional manifold $M^{2}$ with geodesically equivalent Riemannian metrics $g$ and $\bar{g}$ on it. Let $p \in M$ be a point where the metrics are not proportional. Then, $L(g, \bar{g})$ has two (real) eigenvalues $\lambda_{1} \neq \lambda_{2}$ at every point of a small neighborhood $U(p)$. Then, in the notation of Theorem 1.3. $k=2, r=0$ and $m_{1}=m_{2}=1$. Thus, $\chi_{1}=t-\lambda_{1}$, $\chi_{2}=t-\lambda_{2}$ and $\hat{\chi}=\left(t-\lambda_{2}\right)+\left(t-\lambda_{1}\right)$. Then, there exists a coordinate system $x, y$ such that $\lambda_{1}=X(x), \lambda_{2}=Y(y)$ and $h, L$ and $\hat{\chi}(L)$ are given by the matrices

$$
\begin{gathered}
h=\left(\begin{array}{cc}
\tilde{X}(x) & \\
& \tilde{Y}(y)
\end{array}\right), \quad L=\left(\begin{array}{rr}
X(x) & \\
& Y(y)
\end{array}\right), \\
\hat{\chi}(L)=\left(\begin{array}{rl}
Y(y)-X(x) & \\
& X(x)-Y(y)
\end{array}\right) .
\end{gathered}
$$

Combining this with (4), (13), we see that the metrics $g$ and $\bar{g}$ are given by

$$
g=(Y(y)-X(x))\left(\tilde{X}(x) d x^{2}+\tilde{Y}(y) d y^{2}\right)
$$

and

$$
\bar{g}=\left(\frac{1}{X(x)}-\frac{1}{Y(y)}\right)\left(\frac{\tilde{X}(x) d x^{2}}{X(x)}+\frac{\tilde{Y}(y) d y^{2}}{Y(y)}\right) .
$$

By a coordinate change of the form $x_{\text {new }}=x_{\text {new }}(x), y_{\text {new }}=y_{\text {new }}(x)$ one can 'hide' $\tilde{X}$ in $d x^{2}$ and $\tilde{Y}$ in $d y^{2}$ and obtain the metrics of the form (11).

Vocabulary. We call a point $p \in M$ regular if in some neighborhood $U(p)$ of $p$ the Jordan type of $L$ is constant (that is, the number of eigenvalues and Jordan blocks is the same at all points $x \in U(p)$; the sizes of Jordan blocks are assumed to be fixed too, whereas the eigenvalues can, of course, depend on the point). It is easy to see that almost every point of $M$ is regular; that is, the set of regular points is open and dense on the manifold.

Now, applying the Splitting Lemma in the neighborhood of a regular point, we obtain the metrics $h_{i}$ on $2 m_{i}$ or $m_{i}$ dimensional discs and $(1,1)$-tensors $L_{i}$ compatible with $h_{i}$. Moreover, each $L_{i}$ has one real or two complex conjugate eigenvalues, and the Jordan type of $L_{i}$ is the same at all points.

Describing all possible $h_{i}$ and $L_{i}$ satisfying these conditions gives all possible geodesically equivalent metrics $g$ and $\bar{g}$ near regular points: all possible $g$ and $g$ compatible $L$ can be obtained by the gluing construction (which is given by explicit formulas (11), (12) ), and the metric $\bar{g}$ is constructed from $g$ and $L$ by the formula (41). 
Finally, in order to describe the metric and $L$ in the neighborhood of almost any point, it is sufficient to describe the metrics $h_{i}$ and the $h_{i}$-compatible $L_{i}$ such that $L_{i}$ has one real eigenvalue or two complex conjugate eigenvalues, and the type of the Jordan block is the same in the whole neighborhood. We will formulate the result in $\$ 1.3$, see Theorems 1.4, 1.5, 1.9. The proof of these theorems will be given in $\S \$ 2$ and 4 .

1.3. Canonical forms for basic blocks. Throughout this section we assume that $p \in M$ is a regular point; i.e., the Jordan type of $L$ remains unchanged in some neighborhood of $p$. Our goal is to find local normal forms for compatible $L$ and $g$ near $p$ (see Definition 1.1).

According to the previous section (Theorem 1.3), it is sufficient to describe the structure of compatible pairs $(g, L)$ in the case when $L$ either has a single real eigenvalue $\lambda$ or has a pair of complex nonreal eigenvalues $\lambda, \bar{\lambda}$. However even in these cases, the situation depends essentially on the algebraic structure of $L$, more precisely on the geometric multiplicity of $\lambda$, i.e., the number of linearly independent eigenvectors. There are three essentially different possibilities: 1) the geometric multiplicity of $\lambda$ is at least two, 2) $L$ is conjugate to a real Jordan block (i.e., $L$ has a single real eigenvalue $\lambda$ of geometric multiplicity one) and 3 ) $L$ is conjugate to a pair of complex conjugate Jordan blocks (i.e., $L$ has a pair of complex conjugate eigenvalues each of geometric multiplicity one). These cases are described by Theorems 1.4, 1.5 and 1.9 below.

We start with the case of geometric multiplicity $\geq 2$. This situation turns out to be very special. Namely, the following statement holds.

Theorem 1.4. Let $g$ and $L$ be compatible and, in a neighborhood $U$ of a point $p \in M$, let the operator $L$ have either a unique real eigenvalue $\lambda=\lambda(x)$ or $a$ unique pair of complex conjugate eigenvalues $\lambda(x), \bar{\lambda}(x), \operatorname{Im} \lambda(x) \neq 0$. Suppose that the geometric multiplicity of $\lambda$ is at least two at each point $x \in U$. Then the function $\lambda(x)$ is constant and $L$ is covariantly constant in $U$, i.e., $\nabla L=0$. In particular the metrics $g$ and $\bar{g}$ given by (4) are affinely equivalent.

Thus, in the case of geometric multiplicity $\geq 2$, our problem is reduced to another rather nontrivial problem of local classification of pairs $g, L$ satisfying $\nabla L=0$, which has been recently completely solved by Charles Boubel, and we refer to his work [12] for further details (see also [11] for nontrivial examples of such pairs $g$ and $L)$.

We now give the answer for $L$ being conjugate to a single real Jordan block; in other words we assume that the eigenvalue $\lambda$ is real and $L$ possesses a unique (up to proportionality) eigenvector.

Theorem 1.5. Let $g$ and $L$ be compatible and $L$ be conjugate to a single Jordan block with a real eigenvalue $\lambda$. Then there exists a local coordinate system $x_{1}, \ldots, x_{n}$ such that $\lambda$ depends only on $x_{n}$ and that

$$
g=\left(\begin{array}{cccccc} 
& & & & & a_{n-1} \\
& & & 1 & & a_{n-2} \\
& & . & & 0 & \vdots \\
& 1 & & & a_{1} \\
a_{n-1} & a_{n-2} & \ldots & a_{1} & \sum_{i=1}^{n-2} a_{i} a_{n-i-1}
\end{array}\right)
$$


and

$$
L=\left(\begin{array}{ccccc}
\lambda\left(x_{n}\right) & 1 & & & a_{1} \\
& \lambda\left(x_{n}\right) & \ddots & & a_{2} \\
& & \ddots & 1 & \vdots \\
& & & \lambda\left(x_{n}\right) & a_{n-1} \\
& & & & \lambda\left(x_{n}\right)
\end{array}\right)
$$

where

$$
\begin{aligned}
& a_{1}=\lambda_{x_{n}}^{\prime} x_{1}, \\
& a_{2}=2 \lambda_{x_{n}}^{\prime} x_{2}, \\
& \ldots \\
& a_{n-2}=(n-2) \lambda_{x_{n}}^{\prime} x_{n-2}, \\
& a_{n-1}=1+(n-1) \lambda_{x_{n}}^{\prime} x_{n-1},
\end{aligned}
$$

and $\lambda_{x_{n}}^{\prime}$ stands for $\partial \lambda / \partial x_{n}$. Conversely, if $\lambda=\lambda\left(x_{n}\right)$ is an arbitrary smooth function such that $\lambda\left(x_{n}\right) \neq 0$ for all $x_{n}$, then $g$ and $L$ given by the above formulas are compatible (in the domain where $g$ is nondegenerate, i.e. $1+(n-1) \lambda_{x_{n}}^{\prime} x_{n-1} \neq 0$ ).

Remark 1.6. Equivalently, one can write $g$ as the symmetric 2-form

$$
\sum_{k=1}^{n}\left(d x_{k}+(k-1) \lambda_{x_{n}}^{\prime} x_{k-1} d x_{n}\right)\left(d x_{n-k+1}+(n-k) \lambda_{x_{n}}^{\prime} x_{n-k} d x_{n}\right),
$$

with the convention $x_{0}=0$. The $(1,1)$-tensor $L$, in this notation, takes the following form:

$$
L=\lambda\left(x_{n}\right) \cdot \mathbf{1}+\sum_{k=1}^{n-1} \partial_{x_{k}} \otimes d x_{k+1}+\lambda_{x_{n}}^{\prime}\left(\sum_{k=1}^{n-1} k x_{k} \partial_{x_{k}}\right) \otimes d x_{n} .
$$

Remark 1.7. In the case when $\lambda_{x_{n}}^{\prime} \neq 0$ at the point $p$, we can simplify these formulas even further by taking $\lambda\left(x_{n}\right)$ as a new coordinate. After the change $x_{n}^{\text {new }}=\lambda\left(x_{n}\right)$ we obtain the following normal forms for $L$ and $g$ (we keep the "old" notation $x_{n}$ for the "new" coordinate).

Let $g$ and $L$ be compatible and $L$ be conjugate to a Jordan block with a real eigenvalue $\lambda$. If $d \lambda(p) \neq 0$, then in a neighborhood of $p \in M$ there exists a local coordinate system $x_{1}, \ldots, x_{n}$ such that $\lambda=x_{n}, \lambda_{0}=x_{n}(p) \neq 0$, and

$$
g=\left(\begin{array}{cccccc} 
& & & & h\left(x_{n}\right)+(n-1) x_{n-1} \\
& & & 1 & (n-2) x_{n-2} \\
& & . & & 0 & \vdots \\
& 1 & & & & x_{1} \\
h\left(x_{n}\right)+(n-1) x_{n-1} & (n-2) x_{n-2} & \ldots & x_{1} & & \sum
\end{array}\right)
$$

and

$$
L=\left(\begin{array}{ccccc}
x_{n} & 1 & & & x_{1} \\
& x_{n} & \ddots & & 2 x_{2} \\
& & \ddots & 1 & \vdots \\
& & & x_{n} & h\left(x_{n}\right)+(n-1) x_{n-1} \\
& & & & x_{n}
\end{array}\right)
$$


where $\sum=\sum_{i=1}^{n-2} i(n-i+1) x_{i} x_{n-i-1}$ and $h\left(x_{n}\right)$ is an arbitrary function such that $h\left(\lambda_{0}\right) \neq 0$ (equal to $1 / \lambda_{x_{n}^{\text {old }}}^{\prime}$ ). Conversely, $g$ and $L$ given by these formulas are compatible for every $h\left(x_{n}\right)$ (in the domain where $x_{n} \neq 0$ and $h\left(x_{n}\right)+(n-1) x_{n-1} \neq$ $0)$.

Remark 1.8. It follows immediately from the proof (see 93 ) that the canonical coordinate system (and hence canonical forms) for $g$ and $L$ from Theorem 1.5 is uniquely defined (up to a finite group) if we fix the position of the initial point $p \in M$ by saying that coordinates of $p$ in the canonical coordinate system are $\left(0, \ldots, 0, \lambda_{0}\right)$. If we do not fix $p$, i.e., move the origin to another point $p^{\prime} \in U(p)$, then the function $h\left(x_{n}\right)$ playing the role of the parameter for canonical forms (15) and (16) changes. It is not difficult to check that the transformation that preserves the structure of (15) and (16) has the following form:

$$
\begin{aligned}
& \tilde{x}_{n}=x_{n}, \\
& \tilde{x}_{n-1}=x_{n-1}+P\left(x_{n}\right), \\
& \tilde{x}_{n-2}=x_{n-2}-\frac{1}{n-2} P^{\prime}\left(x_{n}\right), \\
& \ldots \\
& \tilde{x}_{n-k}=x_{n-k}+(-1)^{k-1} \frac{1}{(n-2)(n-3) \ldots(n-k)} P^{(k-1)}\left(x_{n}\right), \\
& \ldots \\
& \tilde{x}_{1}=x_{1}+(-1)^{n-2} \frac{1}{(n-2) !} P^{(n-2)}\left(x_{n}\right) .
\end{aligned}
$$

Here $P\left(x_{n}\right)$ is an arbitrary polynomial of degree $n-2$ and $P^{(k)}$ denotes its $k$ th derivative. The function $h\left(x_{n}\right)$ after this change of variables takes the form $h\left(\tilde{x}_{n}\right)-$ $(n-1) P\left(\tilde{x}_{n}\right)$. Thus we see that the function $h$, the parameter of our canonical form, is defined modulo a polynomial of degree $n-2$.

The next case is a complex Jordan block; i.e. we assume that the only eigenvalues of $L$ are a pair of complex conjugate numbers $\lambda$ and $\bar{\lambda}$ for each of which there is a single (up to proportionality) eigenvector over $\mathbb{C}$. Equivalently, this means that the corank of the real operator $(L-\lambda \cdot \mathbf{1})(L-\bar{\lambda} \cdot \mathbf{1})$ is two. In this case, the normal form for $g$ and $L$ can be described in the following way.

Theorem 1.9. Let $g$ and $L$ be compatible and $L$ be conjugate to a complex Jordan block with complex conjugate eigenvalues $\lambda$ and $\bar{\lambda}(\operatorname{Im} \lambda \neq 0)$. Then there exist a complex structure $J$ and a local complex coordinate system $\left(z_{1}, \ldots, z_{n}\right)$ such that

(1) the eigenvalue $\lambda$ is a holomorphic function of $z_{n}$,

(2) $L$ is a complex linear operator on $\left(T_{P} M, J\right)$ given in this coordinate system by the matrix

$$
L^{\mathbb{C}}=\left(\begin{array}{ccccc}
\lambda\left(z_{n}\right) & 1 & & & a_{1} \\
& \lambda\left(z_{n}\right) & \ddots & & a_{2} \\
& & \ddots & 1 & \vdots \\
& & & \lambda\left(z_{n}\right) & a_{n-1} \\
& & & & \lambda\left(z_{n}\right)
\end{array}\right)
$$


(3) the metric $g$ is the real part of the complex bilinear form on $\left(T_{P} M, J\right)$ given in this coordinate system by the matrix

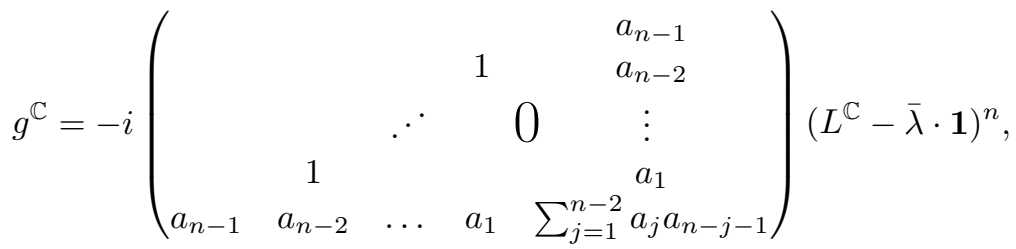

where

$$
\begin{aligned}
& a_{1}=\lambda_{z_{n}}^{\prime} z_{1}, \\
& a_{2}=2 \lambda_{z_{n}}^{\prime} z_{2}, \\
& \ldots \\
& a_{n-2}=(n-2) \lambda_{z_{n}}^{\prime} z_{n-2}, \\
& a_{n-1}=1+(n-1) \lambda_{z_{n}}^{\prime} z_{n-1} .
\end{aligned}
$$

In the real coordinate system $x_{1}, y_{1}, x_{2}, y_{2}, \ldots, x_{n}, y_{n}$ (where $z_{k}=x_{k}+i y_{k}$ ), the operator $L$ and metric $g$ are defined by the $2 n \times 2 n$ real matrices which can be obtained from $L^{\mathbb{C}}$ and $g^{\mathbb{C}}$ by the following standard rule:

- each complex entry $a+i b$ of $L^{\mathbb{C}}$ is replaced by the $2 \times 2$ block $\left(\begin{array}{cc}a & -b \\ b & a\end{array}\right)$,

- each complex entry $a+i b$ of $g^{\mathbb{C}}$ is replaced by the $2 \times 2$ block $\left(\begin{array}{cc}a & -b \\ -b & -a\end{array}\right)$.

Conversely, $g$ and $L$ defined by the above formulas are compatible for every holomorphic function $\lambda\left(z_{n}\right)$ (in the domain where $\lambda\left(z_{n}\right) \neq 0$ and $\operatorname{det} g \neq 0$, i.e., $\left.1+(n-1) \lambda_{z_{n}}^{\prime} z_{n-1} \neq 0\right)$.

As we see, the case of a complex Jordan block is very similar to the real one. However, there is one very essential difference: the additional factor $\left(L^{\mathbb{C}}-\bar{\lambda} \cdot \mathbf{1}\right)^{n}$ in the formula for $g$. Notice, by the way, that unlike $L^{\mathbb{C}}$ the components of $g^{\mathbb{C}}$ are not holomorphic functions on $M$ because of $\bar{\lambda}$ involved in this formula.

Remark 1.10. If the differential of $\lambda$ does not vanish at the point $p$, then just as in the case of a real Jordan block, we can take $\lambda$ as the coordinate $z_{n}$ and obtain a complex version of the canonical forms (15) and (16). The reader can find the explicit formulas in the extended version of this paper in the arXiv [10].

1.4. Perspectives and first global results. It is hard to overestimate the role of the Levi-Civita theorem in the local and global theory of geodesically equivalent Riemannian metrics. Almost all local results are based on it or can easily be proved using it. Though the Levi-Civita theorem is local, most global (i.e., when the manifold is compact) results on geodesically equivalent Riemannian metrics also use the Levi-Civita theorem as an important tool. Roughly speaking, using the Levi-Civita description one can reduce any problem that can be stated using geometric PDEs (for example, any problem involving the curvature) to solve or analyse a system of ODEs.

We expect that our result will play the same role in the pseudo-Riemannian case. We suggest using it to prove the natural generalization of the projective Lichnerowicz-Obata conjecture and the Sophus Lie problem for the pseudoRiemannian case; see [9, §2.2] for the description of the problems. Note that the Lichnerowicz-Obata conjecture was solved, in the Riemannian case, in [43] under 
additional assumptions and in 36] in full generality, and the solution essentially used the Levi-Civita theorem. The Sophus Lie problem was solved in the Riemannian case for dimensions $n>2$ in [43]; the solution again used the Levi-Civita theorem and in the 2-dimensional case for all signatures in [38, where the solution essentially used the description of 2-dimensional projectively equivalent Riemannian and pseudo-Riemannian metrics obtained for example in [8].

We also hope that our description will be helpful for the understanding of the global structure of the manifolds carrying geodesically equivalent pseudoRiemannian metrics. One of the ultimate goals could be to understand the "possible topology" of such manifolds. Though our main theorem is local, it can be effectively used (as was the case with the Levi-Civita theorem) in the global setting too. In particular, we prove the following two results.

Theorem 1.11. Let $M$ be a closed connected manifold. Suppose $g$ and $\bar{g}$ are geodesically equivalent metrics on it and $L$ given by (2) has a complex eigenvalue $\lambda \in \mathbb{C} \backslash \mathbb{R}$ at least at one point. Then at every point of $M$ this number $\lambda$ is an eigenvalue of $L$. Moreover, the multiplicity of the eigenvalue $\lambda$ is the same at every point of the manifold.

Corollary 1.12. Let $M^{3}$ be a closed connected 3-dimensional manifold. Suppose $g$ and $\bar{g}$ are geodesically equivalent metrics on it and at least at one point, L given by (2) has a nonreal eigenvalue. Then $M^{3}$ can be finitely covered by the 3-torus.

\section{Proof of Theorem 1.4; Case of geometric multiplicity $\geq 2$}

We assume that $(M, g)$ is connected and that (a selfadjoint $(1,1)$-tensor) $L$ is compatible with $g$. Our first goal is to prove

Proposition 2.1. Assume that in a neighborhood $U \subseteq M$ there exists a continuous function $\lambda: U \rightarrow \mathbb{R}$ or $\lambda: U \rightarrow \mathbb{C}$ such that for every $x \in U$ the number $\lambda(x)$ is an eigenvalue of $L$ at $x$ of geometric multiplicity at least two. Then, the function $\lambda$ is constant; moreover, for every point $x \in M$ the number $\lambda$ is an eigenvalue of $L$ at $x$ of geometric multiplicity at least two.

Proof. Our proof will use the following theorem due to [6, 33, 44. For any $(1,1)-$ tensor $A$ on $M$, let us denote by $\operatorname{co}(A)^{\top}$ the $(1,1)$-tensor whose matrix in a local coordinate system is the comatrix of (= adjoint matrix to) $A$ transposed. It is indeed a well-defined tensor field: smoothness follows from the fact that the components of $\operatorname{co}(A)^{\top}$ are algebraic expressions in the entries of $A$. The changeof-basis transformation law holds for $\operatorname{co}(A)^{\top}$ if $A$ is nondegenerate, since in this case $\operatorname{co}(A)^{\top}=\operatorname{det}(A) A^{-1}$. As nondegenerate matrices are dense in the set of all quadratic matrices, the transformation law holds for any $A$.

Theorem 2.2. Let $L$ be compatible with $g$. Then for any $t \in \mathbb{R}$, the function

$$
I_{t}: T M \rightarrow \mathbb{R}, \quad I_{t}(\xi)=g\left(\operatorname{co}(L-t \cdot \mathbf{1})^{\top} \xi, \xi\right)
$$

is an integral of the geodesic flow of the metric $g$.

Recall that a function $I$ is an integral if for every geodesic $\gamma$ parameterized by a natural parameter $s$ (such that $\nabla_{\dot{\gamma}} \dot{\gamma}=0$ ), the function $s \mapsto I(\dot{\gamma}(s))$ is constant.

We first consider the case when the function $\lambda$ is real. As the algebraic multiplicity of the eigenvalue $\lambda$ is upper semi-continuous, replacing perhaps $U$ by an 
open subset of it, we may assume that the algebraic multiplicity is constant on $U$. Then, the implicit function theorem easily implies that $\lambda$ is a smooth function.

First we prove that $\lambda$ is constant on $U$. By contradiction, assume that there exists $p \in U$ where the differential of $\lambda$ is not zero. Then, in a small neighborhood of $p$, the set

$$
M_{\lambda(p)}:=\{q \in M \mid \lambda(q)=\lambda(p)\}
$$

is a smooth submanifold of $M$ of codimension 1 . At every point of $M_{\lambda(p)}$, the matrix of the tensor $(L-\lambda(p) \cdot \mathbf{1})$ has rank at most $n-2$, so $\operatorname{co}(L-\lambda(p) \cdot \mathbf{1})^{\top}=\mathbf{0}$. Consequently, for every point $q \in M_{\lambda(p)}$ and for every $\xi \in T_{q} M$ we have $I_{\lambda(p)}(\xi)=0$. Now, take a point $x \in U, x \notin M_{\lambda(p)}$, and consider all geodesics $\gamma_{q, x}$ connecting the points $q \in M_{\lambda(p)}$ with $x$. We assume that the parameter $s$ on the geodesic is natural, $\gamma_{q, x}(0)=q \in M_{\lambda(p)}$ and $\gamma_{q, x}(1)=x$. Since $M_{\lambda(p)}$ has codimension one, for all $x$ that are sufficiently close to $p$, the set of vectors that are proportional to the velocity vectors $\dot{\gamma}_{q, x}(1)$ of such geodesics contains an open nonempty subset of $T_{x} M$. Then, $\operatorname{co}(L-\lambda(p) \cdot \mathbf{1})^{\top}$ is zero at the point $x$. It follows immediately that $\lambda(p)$ is an eigenvalue of $L$ at the point $x$. Then, $\lambda$ is constant in a neighborhood of $p$, which contradicts our assumption that $d \lambda_{\mid p} \neq 0$. The contradiction shows that $\lambda$ is constant on $U$.

Let us now consider the case when $L$ has two complex conjugate eigenvalues $\lambda, \bar{\lambda}: U \subseteq M \rightarrow \mathbb{C}, \operatorname{Im} \lambda \neq 0$. We again assume without loss of generality that the algebraic multiplicity of the eigenvalue $\lambda(x)$ is the same at all points $x \in U$, which in particular implies that $\lambda$ is a smooth function. We first note that for every $(1,1)$-tensor $A$, the $(1,1)$-tensor $\operatorname{co}(A-t \cdot \mathbf{1})^{\top}$ is a polynomial in $t$ of degree $n-1$ whose coefficients are $(1,1)$-tensors. Then, for every complex number $\tau$, the real and imaginary parts of the complex-valued function

$$
I_{\tau}: T M \rightarrow \mathbb{C}, I_{\tau}(\xi):=g\left(\operatorname{co}(L-\tau \cdot \mathbf{1})^{\top} \xi, \xi\right)
$$

are also integrals. Since $\operatorname{rank}(L(q)-\lambda(q) \cdot \mathbf{1}) \leq n-2$, for every $q$ such that $\lambda(q)=\tau$ we have that $I_{\tau}(\xi)=0$ for every $\xi \in T_{q} M$.

Suppose $\lambda$ is not constant on $U$. Then for a certain point $p$ of $U$ its differential is not zero. Suppose first that the differential of the real part of $\lambda$ is proportional to the differential of the imaginary part at all points of a certain neighborhood of $p$. Then, in a sufficiently small neighborhood $U^{\prime}(p) \subseteq U$ of $p$ the set $M_{\lambda(p)}:=$ $\{q \in M \mid \lambda(q)=\lambda(p)\}$ is a submanifold of dimension $n-1$, as it was in the case of a real eigenvalue $\lambda$. Then, repeating the same arguments as above, we conclude that $\lambda(x)=\lambda(p)$ for all $x$ from a small neighborhood of $p$, which gives us a contradiction with the assumption that the differential of $\lambda$ does not vanish at $p$. The contradiction shows that $\lambda$ is a constant provided the differential of the real part of $\lambda$ is proportional to the differential of the imaginary part in some $U^{\prime} \subseteq U$.

Let us now suppose that the differential of the real part of $\lambda$ at the point $p$ is not proportional to the differential of the imaginary part. Then, the set $M_{\lambda(p)}:=$ $\{q \in M \mid \lambda(q)=\lambda(p)\}$ is (in a sufficiently small neighborhood $U^{\prime}(p) \subseteq U$ ) a submanifold of dimension $n-2$. We again take an arbitrary point $x$ that is sufficiently close to $p$ and consider all geodesics $\gamma_{q, x}$ connecting the points $q \in M_{\lambda(p)}$ with $x$ assuming as above that $\gamma_{q, x}(0) \in M_{\lambda(p)}$ and $\gamma_{q, x}(1)=x$. The set of the tangent vectors at $x$ that are proportional to the velocity vectors to such geodesics at the point $x$ contains a submanifold of codimension 1 of $T_{x} M$, implying that the real part of $I_{\lambda(p)}(\xi)$ is proportional to the imaginary part of $I_{\lambda(p)}(\xi)$ for all $\xi \in T_{x} M$ (the 
coefficient of the proportionality is a constant on each $T_{x} M$ but may a priori depend on $x$ ). Now, since the real and the imaginary parts of $I_{\lambda(p)}$ are both integrals, the coefficient of proportionality of these functions is an integral too, implying it is constant. Then, for a certain complex constant $a+i b \neq 0$, for every $x \in U^{\prime}(p)$ and every $\xi \in T_{x} M$ we have $(a+i b) I_{\lambda(p)}(\xi)=(a-i b) I_{\bar{\lambda}(p)}(\xi)$ so that

$$
(a+i b) \operatorname{co}(L-\lambda(p) \cdot \mathbf{1})^{\top}=(a-i b) \operatorname{co}(L-\bar{\lambda}(p) \cdot \mathbf{1})^{\top} .
$$

For points $x$ such that $\lambda(x) \neq \lambda(p)$ the matrix $L-\lambda(p) \cdot \mathbf{1}$ is nondegenerate, and (18) implies that $(L-\lambda(p) \cdot \mathbf{1})^{-1}$ is proportional to $(L-\bar{\lambda}(p) \cdot \mathbf{1})^{-1}$. Hence, $L-\lambda(p) \cdot \mathbf{1}$ and $L-\bar{\lambda}(p) \cdot \mathbf{1}$ are proportional too, which contradicts the assumption that $\lambda$ is not real. The contradiction shows that at all points of the neighborhood $U$ (such that $\lambda(x)$ is an eigenvalue of $L$ of geometric multiplicity at least two at every point $x$ ) the function $\lambda$ is a constant.

Let us now show that this (real or not) constant $\lambda$ is an eigenvalue of $L$ of geometric multiplicity at least two at every point of the whole of $M$. We first consider a point $p \in M \backslash U$ that can be connected with a point of $U$ by a geodesic $\gamma$, where $U$ is a neighborhood such that at each of its points $L$ has (constant) eigenvalue $\lambda$ of multiplicity at least two; we assume that $\gamma(0)=p$ and $\gamma(1) \in U$. We consider a small open neighborhood $V \subseteq T_{p} M$ of $\xi=\dot{\gamma}(0)$. If $V$ is sufficiently small, for every $\eta \in V$ the point $\gamma_{p, \eta}(1)$ of the geodesic $\gamma_{p, \eta}$ such that $\gamma(0)=p$ and $\dot{\gamma}(0)=\eta$ lies in $U$. Since at each point of $U$ the constant $\lambda$ is an eigenvalue of $L$ of multiplicity at least two, $I_{\lambda}\left(\dot{\gamma}_{p, \eta}(1)\right)=0$, implying $I_{\lambda}\left(\dot{\gamma}_{p, \eta}(0)\right)=0$. Hence, $I_{\lambda} \equiv 0$ on an open nonempty subset of $T_{p} M$ implying $I_{\lambda} \equiv 0$ on the whole $T_{p} M$ so $\lambda$ is an eigenvalue of $L$ at $p$ of multiplicity at least two. Now, if $p$ can be connected by a geodesic with a point of $U$, then any point from a sufficiently small neighborhood of $p$ can also be connected by a geodesic with a point of $U$, so $\lambda$ is an eigenvalue of $L$ of multiplicity at least two at every point of a small neighborhood of $p$. To come to the same conclusion on the whole of $M$, it suffices to notice that every point $x \in M$ can be joined with $U$ by a piecewise smooth curve such that each smooth segment of it is a geodesic. Proposition 2.1 is proved.

Corollary 2.3. In the hypotheses of Proposition 2.1 , assume in addition that $\lambda \in \mathbb{R}$ is the unique eigenvalue of $L$ (or $\lambda, \bar{\lambda} \in \mathbb{C} \backslash \mathbb{R}$ are the unique eigenvalues of $L$ ); then $L$ is covariantly constant, i.e., $\nabla L=0$, or equivalently, $g$ and $\bar{g}$ are affinely equivalent.

The proof is obvious: since $\lambda$ is constant, so is $\operatorname{tr} L$. Hence, the right hand side of (5) vanishes and we get $\nabla L=0$.

Thus, if the eigenspace of $L$ has dimension $\geq 2$, then our problem is reduced to the classification of pairs of affinely equivalent pseudo-Riemannian metrics, which has been recently obtained by Boubel in 12 .

\section{Proof of Theorem 1.5: CASe of a ReAl Jordan Block}

3.1. Canonical frames and uniqueness lemma. Let $L$ be a $g$-selfadjoint operator on a real vector space $V$. It is a natural question to ask to which canonical form we can simultaneously reduce (the matrices of) $L$ and $g$ by an appropriate change of a basis. The answer is well known (see, for example, [28, [29]) and is given by the following. 
Proposition 3.1. There exists a canonical basis $e_{1}, \ldots, e_{n} \in V$ in which $L$ and $g$ can be simultaneously reduced to the following block diagonal canonical forms:

$$
L_{\text {can }}=\left(\begin{array}{llll}
L_{1} & & & \\
& L_{2} & & \\
& & \ddots & \\
& & & L_{s}
\end{array}\right), \quad g_{\text {can }}=\left(\begin{array}{llll}
g_{1} & & & \\
& g_{2} & & \\
& & \ddots & \\
& & & g_{s}
\end{array}\right) \text {, }
$$

where

$$
L_{j}=\left(\begin{array}{cccc}
\lambda & 1 & & \\
& \lambda & \ddots & \\
& & \ddots & 1 \\
& & & \lambda
\end{array}\right)
$$

in the case of a real eigenvalue $\lambda \in \mathbb{R}$ (real Jordan block) or

$$
L_{j}=\left(\begin{array}{rrrrrrr}
a & -b & 1 & 0 & & & \\
b & a & 0 & 1 & & & \\
& & a & -b & \ddots & & \\
& & b & a & & & \\
& & & & \ddots & 1 & 0 \\
& & & & & 0 & 1 \\
& & & & & a & -b \\
& & & & & b & a
\end{array}\right)
$$

in the case of complex conjugate eigenvalues $\lambda_{1,2}=a \pm i b, b \neq 0$ (complex Jordan block), and

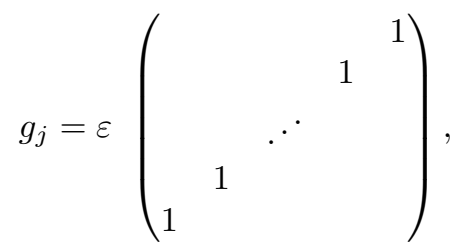

where $\varepsilon= \pm 1$ in the case $\lambda \in \mathbb{R}$ and $\varepsilon=1$ for $\lambda_{1,2}=a \pm i b \in \mathbb{C} \backslash \mathbb{R}$. It is assumed that for each $j$ the blocks $g_{j}$ and $L_{j}$ are of the same size and that the corresponding eigenvalues depend on $j$.

Remark 3.2. Notice that the canonical forms $g_{\text {can }}$ and $L_{\text {can }}$ can be chosen in many different ways. For example, in the complex case we can replace $g_{\text {can }}$ by $g_{\text {can }} P\left(L_{\text {can }}\right)$ where $P(t)$ is an arbitrary polynomial such that $P\left(L_{\text {can }}\right)$ is invertible. Indeed, as $L_{\text {can }}$ is selfadjoint w.r.t. $g_{\text {can }}$, the pair $\left(g_{\text {can }} P\left(L_{\text {can }}\right), L_{\text {can }}\right)$ is also a pair consisting of a nondegenerate symmetric bilinear form and a selfadjoint operator w.r.t. it. Moreover, as $L_{\text {can }}$ is "complex", it follows from Proposition 3.1 that $\left(g_{\text {can }}, L_{\text {can }}\right)$ and $\left(g_{\text {can }} P\left(L_{\text {can }}\right), L_{\text {can }}\right)$ are conjugate to each other, since in the "complex" case the canonical form for $g$ is uniquely defined by $L$. For our purposes, by a canonical form of $L$ and $g$ it is convenient to understand any forms where the entries of $L_{\text {can }}$ and $g_{\text {can }}$ depend on the eigenvalues of $L$ only.

Now let $g$ be a pseudo-Riemannian metric on a smooth manifold $M$ and $L$ be a $g$-selfadjoint $(1,1)$-tensor field. Assume that $L$ is regular at each point of a small neighborhood $U(p)$ of a point $p \in M$. Recall that the regularity of $L$ means that 
each eigenvalue of $L$ is of geometric multiplicity one or, equivalently, the Jordan normal form contains exactly one Jordan block for each eigenvalue. This condition implies that the eigenvalues of $L$ are smooth functions on $U(p)$ and the Jordan type of $L$ does not change in $U(p)$; in particular, $p$ is a regular point (see page 6725). In such a situation we can choose smooth linearly independent vector fields $e_{1}, \ldots, e_{n} \in T_{x} M$ in which $L$ and $g$ both take canonical forms. For a regular $L$, these vectors are uniquely defined (up to a discrete group), and we will say that $e_{1}, \ldots, e_{n}$ is a canonical moving frame for $L$ and $g$.

In general, the vector fields $e_{1}, \ldots, e_{n}$ do not commute. To reconstruct a canonical coordinate system on $U(p)$ we need to analyse the commutation relations between them. It turns out that these relations can be obtained from the compatibility equation (5).

Lemma 3.3. Let $e_{1}, \ldots, e_{n}$ be a canonical moving frame for $L$ and $g$ in a neighborhood of a regular point $p \in M$. If $L$ and $g$ are compatible and $L$ is regular, then the covariant derivatives $\nabla_{e_{i}} e_{j}$ and hence the commutators $\left[e_{i}, e_{j}\right]$ can be uniquely expressed as certain linear combinations of $e_{l}$ with the coefficients being functions of $\lambda_{r}$ and their derivatives $e_{s}\left(\lambda_{r}\right)$ along $e_{s}$, where $\lambda_{r}$ are eigenvalues of $L$.

Proof. For the frame $e_{1}, \ldots, e_{n}$ we introduce $B_{u}$ to be a $(1,1)$-tensor field defined by

$$
B_{u} v=\nabla_{u} v
$$

where $u$ and $v$ are vector fields with constant coordinates w.r.t. the frame.

Clearly, $B_{u}$ defines the Levi-Civita connection in the frame $e_{1}, \ldots, e_{n}$, and our goal is to reconstruct it from the compatibility equation (5). The covariant derivative of $L$ in terms of $B_{u}$ can be written as

$$
\nabla_{u} L=\mathcal{D}_{u}(L)+\left[B_{u}, L\right],
$$

where $\mathcal{D}_{u} L$ denotes the operator obtained by differentiating $L$ componentwise along $u=u^{k} e_{k}$; i.e., for $L=L_{j}^{i} e_{i} \otimes e^{j}$, we have $\mathcal{D}_{u} L=u^{k} e_{k}\left(L_{j}^{i}\right) e_{i} \otimes e^{j}$.

To find $B_{u}$, it is convenient to rewrite the compatibility equation in the form

$$
\left[B_{u}, L\right]=\frac{1}{2}\left(u \otimes d \operatorname{tr} L+(u \otimes d \operatorname{tr} L)^{*}\right)-\mathcal{D}_{u} L .
$$

In addition to that we have

$$
\mathcal{D}_{u}\left(g\left(e_{i}, e_{j}\right)\right)=\nabla_{u}\left(g\left(e_{i}, e_{j}\right)\right)=g\left(B_{u} e_{i}, e_{j}\right)+g\left(e_{i}, B_{u} e_{j}\right)
$$

or, equivalently,

$$
B_{u}+B_{u}^{*}=g^{-1} \mathcal{D}_{u} g
$$

Thus, $B_{u}$ satisfies two equations of the form

$$
\begin{aligned}
& {\left[B_{u}, L\right]=C,} \\
& B_{u}+B_{u}^{*}=D,
\end{aligned}
$$

where $C$ and $D$ are certain operators (whose components w.r.t. the moving frame are functions of the eigenvalues $\lambda_{r}$ and their derivatives $e_{s}\left(\lambda_{r}\right)$ ).

The uniqueness of the solution (if it exists!) is a purely algebraic fact. Indeed, consider the corresponding homogeneous system

$$
\begin{aligned}
& {\left[B_{u}, L\right]=0,} \\
& B_{u}+B_{u}^{*}=0 .
\end{aligned}
$$


The first equation means that $B_{u}$ commutes with $L$, i.e., belongs to the centralizer of $L$. Since $L$ is regular, its centralizer is generated by powers of $L$, i.e., $\mathbf{1}, L, L^{2}, \ldots, L^{n-1}$. It follows from this that $B_{u}$ is $g$-selfadjoint. But then the second equation can be rewritten simply as $2 B_{u}=0$. Thus, the homogeneous system has only the trivial solution, which proves the statement.

The commutators $\left[e_{i}, e_{j}\right]$ can now be uniquely reconstructed by means of the standard formula: $\left[e_{i}, e_{j}\right]=\nabla_{e_{i}} e_{j}-\nabla_{e_{j}} e_{i}=B_{e_{i}} e_{j}-B_{e_{j}} e_{i}$.

In the next section we show how the commutation relations between the elements of the canonical moving frame can be found in practice.

Notice that Lemma 3.3 does not say that (24) is always consistent for every $C$ and $D$. In fact, these matrices have to satisfy some additional relations (for example, $\operatorname{tr} C L^{k}=0$ ). These equations, in particular, imply the vanishing of the Nijenhuis torsion of $L$ and, therefore, the fact that $e_{s}\left(\lambda_{r}\right)=0$ for those $e_{s}$ which "do not belong" to the $\lambda_{r}$-block. Another condition of this kind is discussed below in Lemma 3.4.

\subsection{Canonical frame and canonical coordinate system for a real Jordan}

block. Let $L$ be conjugate to a Jordan block with a real eigenvalue. Then we can choose a moving frame $e_{1}, \ldots, e_{n}$ in which $L$ and $g$ take the following canonical forms:

$$
L_{\text {can }}=\left(\begin{array}{cccc}
\lambda(x) & 1 & & \\
& \lambda(x) & \ddots & \\
& & \ddots & 1 \\
& & & \lambda(x)
\end{array}\right), \quad g_{\text {can }}= \pm\left(\begin{array}{ccc} 
& & \\
& 1 & \\
& . & \\
1 & &
\end{array}\right)
$$

Here we apply the ideas from 33.1 to describe the commutation relations between $e_{1}, \ldots, e_{n}$ and then to solve them in order to construct a canonical coordinate system for the pair $g$ and $L$.

As usual, it is convenient to decompose $L$ canonically into the semisimple and nilpotent parts:

$$
L=\lambda(x) \cdot \mathbf{1}+N
$$

Obviously, $N$ is selfadjoint with respect to $g$. The compatibility equation can naturally be rewritten in terms of $N$ :

or

$$
\nabla_{u}(L)=u(\lambda) \cdot \mathbf{1}+\nabla_{u} N=\frac{n}{2}\left(u \otimes d \lambda+(u \otimes d \lambda)^{*}\right)
$$

$$
\left[B_{u}, N\right]=\frac{n}{2}\left(u \otimes d \lambda+(u \otimes d \lambda)^{*}\right)-u(\lambda) \cdot \mathbf{1},
$$

where $B_{u}$, as before, is defined by (22), and we use the fact that the components of $N$ in the frame are all constants so that $\nabla_{u} N=\left[B_{u}, N\right]$. This equation implies

Lemma 3.4. We have $e_{i}(\lambda)=0$, for $i=1, \ldots, n-1$.

Proof. This property is well known for $L$ with zero Nijenhuis torsion (for $L$ this condition is fulfilled; see e.g. 6, Theorem 1]). However, we can easily derive this fact from (27). Indeed, multiply both sides of this equation by $N$ and take the trace. For the left hand side we get

$$
\operatorname{tr}\left(N \cdot\left[B_{u}, N\right]\right)=0 .
$$


For the right hand side:

$\operatorname{tr}\left(N \cdot\left(\frac{n}{2}\left(u \otimes d \lambda+(u \otimes d \lambda)^{*}\right)-u(\lambda) \cdot \mathbf{1}\right)\right)=n \cdot N u(\lambda)-u(\lambda) \cdot \operatorname{tr} N=n \cdot N u(\lambda)$.

Hence, for any vector $v=N u \in \operatorname{Im} N$, we have $v(\lambda)=0$. It remains to notice that $\operatorname{Im} N=\operatorname{span}\left(e_{1}, \ldots, e_{n-1}\right)$.

Thus, in our basis $d \lambda=\left(0, \ldots, 0, e_{n}(\lambda)\right)$. This allows us to get the following explicit form for the right hand side of (27):

$$
\nabla_{u} N=\left[B_{u}, N\right]=e_{n}(\lambda)\left(\begin{array}{cccccc}
\frac{n-2}{2} u_{n} & \frac{n}{2} u_{n-1} & \frac{n}{2} u_{n-2} & \cdots & \frac{n}{2} u_{2} & n u_{1} \\
& -u_{n} & & & \frac{n}{2} u_{2} \\
& & -u_{n} & 0 & \frac{n}{2} u_{3} \\
& & & \ddots & \vdots \\
& & & & -u_{n} & \frac{n}{2} u_{n-1} \\
& & & & & \frac{n-2}{2} u_{n}
\end{array}\right) .
$$

According to Lemma 3.3, page 6734, the solution of this equation is unique. We just give the final answer (the reader can check this result by substituting (29) into $(28)$ ):

$$
B_{u}=e_{n}(\lambda)\left(\begin{array}{cccccc}
\frac{n}{2} u_{n-1} & \frac{n}{2} u_{n-2} & \ldots & \frac{n}{2} u_{2} & \frac{n}{2} u_{1} & 0 \\
\left(1-\frac{n}{2}\right) u_{n} & & & & & -\frac{n}{2} u_{1} \\
& \left(2-\frac{n}{2}\right) u_{n} & & & & -\frac{n}{2} u_{2} \\
& & \ddots & & 0 & \vdots \\
& & & \left(\frac{n}{2}-2\right) u_{n} & & -\frac{n}{2} u_{n-2} \\
& & & & \left(\frac{n}{2}-1\right) u_{n} & -\frac{n}{2} u_{n-1}
\end{array}\right) .
$$

The next step is to find pairwise commutators $\left[e_{i}, e_{j}\right]$.

Lemma 3.5. The vector fields $e_{1}, \ldots, e_{n-1}$ commute.

Proof. Let $u=u_{1} e_{1}+\cdots+u_{n} e_{n}$. It follows from (29) that

$$
\nabla_{u} e_{j}=e_{n}(\lambda)\left(\frac{n}{2} u_{n-j} e_{1}+\left(j-\frac{n}{2}\right) u_{n} e_{j+1}\right), \quad j<n .
$$

Hence, for $i<n, \nabla_{e_{i}} e_{j}=e_{n}(\lambda) \frac{n}{2} e_{1}$ if and only if $i+j=n$; otherwise $\nabla_{e_{i}} e_{j}=0$. In any case

$$
\left[e_{i}, e_{j}\right]=\nabla_{e_{i}} e_{j}-\nabla_{e_{j}} e_{i}=0
$$

for $i, j<n$.

It remains to find the commutators $\left[e_{i}, e_{n}\right]$.

Lemma 3.6. For $i=1, \ldots, n-1$, we have

$$
\left[e_{i}, e_{n}\right]=-i e_{n}(\lambda) \cdot e_{i+1} .
$$

Proof. From (29) we have

$$
\nabla_{e_{i}} e_{n}=-\frac{n}{2} e_{n}(\lambda) \cdot e_{i+1} \quad \text { and } \quad \nabla_{e_{n}} e_{i}=-\left(i-\frac{n}{2}\right) e_{n}(\lambda) \cdot e_{i+1} .
$$

Thus,

$$
\left[e_{i}, e_{n}\right]=-e_{n}(\lambda)\left(\frac{n}{2} e_{i+1}+-\left(i-\frac{n}{2}\right) e_{i+1}\right)=-i e_{n}(\lambda) \cdot e_{i+1},
$$

as stated. 
Our goal now is to find a coordinate system with respect to which $N$ and $g$ have the simplest form. Since the vector fields $e_{1}, \ldots, e_{n-1}$ commute we can choose a coordinate system $x_{1}, \ldots, x_{n}$ in such a way that $e_{1}=\partial_{x_{1}}, \ldots, e_{n-1}=\partial_{x_{n-1}}$. To make our choice unambiguous, we assume that our initial point $p \in M$ has all coordinates zero and, in addition,

$$
e_{n}=\partial_{x_{n}} \quad \text { on the } x_{n} \text {-axes, }
$$

i.e. on the curve $x_{1}=x_{2}=\cdots=x_{n-1}=0$. Notice that the foliation generated by $\operatorname{Im} N$ is given by $x_{n}=$ const and the eigenvalue $\lambda$ depends on $x_{n}$ only.

To rewrite $L$ and $g$ in this coordinate system we just need to find the transition matrix between $e_{1}, \ldots, e_{n}$ and $\partial_{x_{1}}, \ldots, \partial_{x_{n}}$. Since $\partial_{x_{i}}=e_{i}, i=1, \ldots, n-1$, it remains to determine the coefficients (yet unknown) of the linear combination

$$
\partial_{x_{n}}=a_{0} e_{1}+\cdots+a_{n-1} e_{n} .
$$

First we use the fact that $\lambda$ does not depend on $x_{1}, \ldots, x_{n-1}$. Therefore

$$
\lambda_{x_{n}}^{\prime}=\partial_{x_{n}}(\lambda)=\left(a_{0} e_{1}+\cdots+a_{n-1} e_{n}\right)(\lambda)=a_{n-1} e_{n}(\lambda) .
$$

Since $\partial_{x_{n}}$ must commute with each $e_{i}=\partial_{x_{i}}(i<n)$, we obtain a system of differential equations on $a_{j}$ :

$$
\begin{aligned}
0 & =\left[e_{i}, a_{0} e_{1}+\cdots+a_{n-1} e_{n}\right]=\sum_{l=1}^{n} \frac{\partial a_{l-1}}{\partial x_{i}} \cdot e_{l}-a_{n-1} i e_{n}(\lambda) \cdot e_{i+1} \\
& =\sum_{l=1}^{n} \frac{\partial a_{l-1}}{\partial x_{i}} \cdot e_{l}-i \lambda_{x_{n}}^{\prime} \cdot e_{i+1}
\end{aligned}
$$

or, equivalently,

$$
\frac{\partial a_{l-1}}{\partial x_{i}}=0 \quad \text { if } \quad l \neq i+1 \quad \text { and } \quad \frac{\partial a_{i}}{\partial x_{i}}=i \lambda_{x_{n}}^{\prime}, \quad i=1, \ldots, n-1 .
$$

In other words, $a_{0}=a_{0}\left(x_{n}\right)$, whereas $a_{i}$ depends on $x_{i}$ and $x_{n}$ and satisfies the equation

$$
\frac{\partial a_{i}}{\partial x_{i}}=i \lambda_{x_{n}}^{\prime}, \quad i=1, \ldots, n-1,
$$

which can easily be solved. Its general solution is

$$
a_{i}\left(x_{i}, x_{n}\right)=i \lambda_{x_{n}}^{\prime} x_{i}+f_{i}\left(x_{n}\right),
$$

where $f_{i}\left(x_{n}\right)$ is an arbitrary function. But we have a kind of initial condition (30) that requires

$$
a_{i}\left(0, \ldots, 0, x_{n}\right)=0 \quad \text { for } i \neq n-1 \quad \text { and } \quad a_{n-1}\left(0, \ldots, 0, x_{n}\right)=1 .
$$

It follows immediately from this that

$$
\begin{aligned}
& a_{0}=0, \\
& a_{i}=i \lambda_{x_{n}}^{\prime} x_{i}, \quad i=1, \ldots, n-2,
\end{aligned}
$$

and

$$
a_{n-1}=(n-1) \lambda_{x_{n}}^{\prime} x_{n-1}+1
$$


Thus, the transition matrix $C$ has been found:

$$
\left(\partial_{x_{1}}, \ldots, \partial_{x_{n}}\right)=\left(e_{1}, \ldots, e_{n}\right) \cdot C, \quad \text { with } C=\left(\begin{array}{cccc}
1 & & & a_{0} \\
& 1 & & a_{1} \\
& & \ddots & \vdots \\
& & & a_{n-1}
\end{array}\right)
$$

and $a_{0}, \ldots, a_{n-1}$ defined by (31) and (32).

Now to obtain the form of $g$ and $L$ in the local coordinates $x_{1}, \ldots, x_{n}$, we only need to apply the standard rule

$$
L_{\text {can }} \longrightarrow L=C^{-1} L_{\text {can }} C, \quad g_{\text {can }} \longrightarrow g=C^{\top} g_{\text {can }} C,
$$

where $L_{\text {can }}$ and $g_{\text {can }}$ are defined by (26). A straightforward computation of $L$ and $g$ gives the statement of Theorem 1.5 , page 6726 .

The converse statement easily follows from direct verification (also one may notice that the above arguments are, in fact, invertible and therefore $L$ and $g$ given by Theorem 1.5 satisfy the compatibility equation (5) automatically).

\section{Proof of Theorem 1.9; \\ A PAIR OF COMPLEX CONJUGATE JORDAN BLOCKS}

In this section we assume that $L$ has two complex conjugate eigenvalues $\lambda=a+i b$ and $\bar{\lambda}=a-i b, b \neq 0$ (each of geometric multiplicity one), so that $L$ and $g$ can be simultaneously reduced to the following canonical forms:

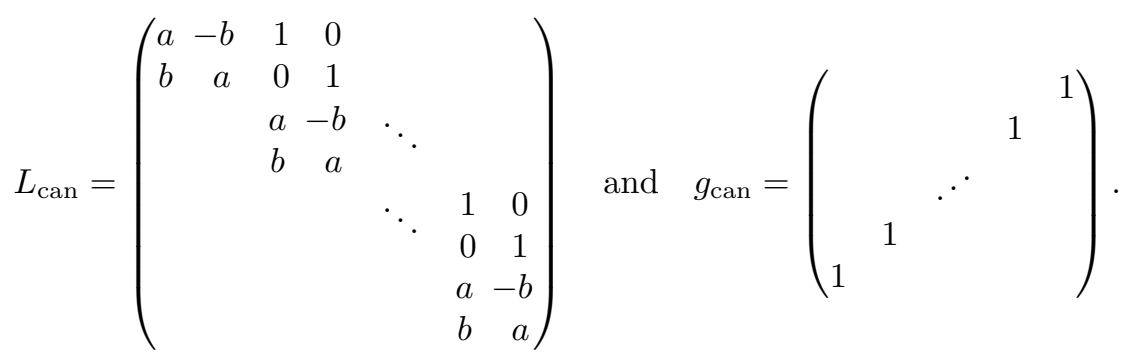

By using the "moving frame" machinery as above, we can find the commutation relations between the elements of the canonical frame (associated with the canonical forms (33) of $L$ and $g$ ) and describe the corresponding canonical coordinate system. However, this approach leads to serious technical difficulties because the commutation relations turn out to be quite complicated. To simplify them we will change the canonical forms of $L$ and $g$ in a certain way which is, in fact, motivated by the splitting construction from [9], which we recalled in $\$ 1.2$ Namely, we set, using Remark 3.2, page 6733 with $P(t)=(t-\lambda)^{n}+(t-\bar{\lambda})^{n}$ :

$$
L_{\text {can }}=L_{\text {can }}^{\text {old }}, \quad g_{\text {can }}=g_{\text {can }}^{\text {old }}\left(\left(L_{\text {can }}^{\text {old }}-\lambda \cdot \mathbf{1}\right)^{n}+\left(L_{\text {can }}^{\text {old }}-\bar{\lambda} \cdot \mathbf{1}\right)^{n}\right),
$$

where $L_{\text {can }}^{\text {old }}$ and $g_{\text {can }}^{\text {old }}$ are as in (33) and $n=\frac{1}{2} \operatorname{dim} M$. Notice that the operator $\left((L-\lambda \cdot \mathbf{1})^{n}+(L-\bar{\lambda} \cdot \mathbf{1})^{n}\right)$ is real, so $g_{\text {can }}$ is a real symmetric matrix.

Let $e_{1}, f_{1}, e_{2}, f_{2}, \ldots, e_{n}, f_{n}$ be the canonical frame associated with these (real) canonical forms (34). To simplify the commutation relations between them, we need one more modification. Namely, we pass from $e_{i}, f_{i}(i=1, \ldots, n)$ to the natural complex frame $\xi_{1}, \ldots, \xi_{n}, \eta_{1}, \ldots, \eta_{n}$ by putting

$$
\xi_{k}=\frac{1}{2}\left(e_{k}-i f_{k}\right) \quad \text { and } \quad \eta_{k}=\frac{1}{2}\left(e_{k}+i f_{k}\right)=\bar{\xi}_{k} .
$$


Thus, from now on we allow ourselves to use formal complex combinations of tangent vectors; i.e., we pass from the real tangent bundle $T M$ to its complexification $T^{\mathbb{C}} M$. In particular, we consider the complex vector fields $\xi=e+i f$, $e, f \in \Gamma(T M)$, and treat them as differential operators on the space of complexvalued smooth functions $w(x)=u(x)+i v(x)$ on $M$ :

$$
\xi(w)=(e(u)-f(v))+i(e(v)+f(u)) .
$$

The commutators of complex-valued vector fields and other objects of this kind are defined in the natural way.

According to Lemma 3.3, we now can uniquely reconstruct the commutation relations between the elements of the frame $\xi_{1}, \ldots, \xi_{n}, \eta_{1}, \ldots, \eta_{n}$ and information about derivatives of $\lambda$ and $\bar{\lambda}$ along these elements. Here is the result.

Proposition 4.1. Let $e_{1}, f_{1}, e_{2}, f_{2}, \ldots, e_{n}, f_{n}$ be the canonical frame associated with canonical forms (34). Then the complex frame

$$
\xi_{1}, \ldots, \xi_{n}, \eta_{1}, \ldots, \eta_{n}
$$

defined by (35) satisfies the following properties:

(1) $\xi_{k}$ and $\eta_{m}$ commute for all $k, m$;

(2) $\xi_{1}, \ldots, \xi_{n-1}$ commute and $\eta_{1}, \ldots, \eta_{n-1}$ commute (in particular, all real vector fields $e_{k}$ and $f_{m}$ commute for all $\left.k, m \leq n-1\right)$;

(3) the only nonzero derivatives are $\xi_{n}(\lambda)$ and $\eta_{n}(\bar{\lambda})$;

(4) nontrivial commutation relations are:

$$
\begin{array}{ll}
{\left[\xi_{1}, \xi_{n}\right]=-\xi_{n}(\lambda) \cdot \xi_{2},} & {\left[\eta_{1}, \eta_{n}\right]=-\eta_{n}(\bar{\lambda}) \cdot \eta_{2},} \\
{\left[\xi_{2}, \xi_{n}\right]=-2 \xi_{n}(\lambda) \cdot \xi_{3},} & {\left[\eta_{2}, \eta_{n}\right]=-2 \eta_{n}(\bar{\lambda}) \cdot \eta_{3},} \\
{\left[\xi_{3}, \xi_{n}\right]=-3 \xi_{n}(\lambda) \cdot \xi_{4},} & {\left[\eta_{3}, \eta_{n}\right]=-3 \eta_{n}(\bar{\lambda}) \cdot \eta_{4},} \\
\ldots & \ldots \\
{\left[\xi_{n-1}, \xi_{n}\right]=-(n-1) \xi_{n}(\lambda) \cdot \xi_{n},} & {\left[\eta_{n-1}, \eta_{n}\right]=-(n-1) \eta_{n}(\bar{\lambda}) \cdot \eta_{n} .}
\end{array}
$$

Proof. One can find these relations by straightforward (linear-algebraic) computation, but we shall give another proof based on the splitting construction (see \$1.2) and the uniqueness lemma (Lemma 3.3 , page 6734).

Before discussing the case of a complex Jordan block (more precisely, of two complex conjugate blocks), consider the case of two real Jordan blocks with distinct eigenvalues as an illustrating example. Take two compatible pairs $\left(g_{1}, L_{1}\right)$ and $\left(g_{2}, L_{2}\right)$, each of which represents a single Jordan block with eigenvalue $\lambda_{i} \in \mathbb{R}$ (see the previous section for the complete description). Let $\xi_{1}, \ldots, \xi_{n}$ be the canonical frame for the first pair $\left(g_{1}, L_{1}\right)$ and $\eta_{1}, \ldots, \eta_{k}$ for the second one $\left(g_{2}, L_{2}\right)$. The gluing lemma (Theorem 1.2 page 6722) allows us to construct a new compatible pair $L, g$ by putting

$$
L=\left(\begin{array}{cc}
L_{1} & 0 \\
0 & L_{2}
\end{array}\right), \quad g=\left(\begin{array}{cc}
g_{1} \chi_{2}\left(L_{1}\right) & 0 \\
0 & g_{2} \chi_{1}\left(L_{2}\right)
\end{array}\right)=\left(\begin{array}{cc}
g_{1} & 0 \\
0 & g_{2}
\end{array}\right)\left(\chi_{1}(L)+\chi_{2}(L)\right)
$$

where $\chi_{i}(t)$ is the characteristic polynomial of $L_{i}$. The compatibility of $L_{i}$ and $g_{i}$, $i=1,2$, guarantees the compatibility of $L$ and $g$.

Now we ask ourselves the converse question. Let $\xi_{1}, \ldots, \xi_{n}, \eta_{1}, \ldots, \eta_{k}$ be the canonical frame for a compatible pair $g, L$ having the (nonstandard) canonical form (36) with $L_{i}, g_{i}$ being the standard canonical forms as in (26). What are the commutation relations between the elements of the frame and the conditions on 
the derivatives of the eigenvalues $\lambda_{1}$ and $\lambda_{2}$ along the frame? We mean, of course, those relations which can be derived from the compatibility equation for $g$ and $L$.

Using the uniqueness result (Lemma 3.3), we immediately conclude that these relations will be exactly the same as for two separate Jordan blocks; namely, $\xi_{i}$ 's commute with $\eta_{j}$ 's and the relations within each of these two groups will be those given in Lemmas 3.5 and 3.6 in 33.2

We now notice that in this construction nothing changes if we allow $\lambda_{1}$ and $\lambda_{2}$ to be complex conjugate, i.e., $\lambda_{1}=\lambda$ and $\lambda_{2}=\bar{\lambda}$ with $\operatorname{Im} \lambda \neq 0$. The elements $\xi_{1}, \ldots, \xi_{n}, \eta_{1}, \ldots, \eta_{k}$ of the canonical frame will be, of course, vectors of the complexified tangent space $\left(T_{P} M\right)^{\mathbb{C}}$. The point is that Lemmas 3.3. 3.4, 3.5 and 3.6 are of purely algebraic nature and therefore can be applied to complexified objects without any change.

If $e_{1}, f_{1}, e_{2}, f_{2}, \ldots, e_{n}, f_{n}$ is the canonical frame associated with the (real) canonical forms (34), then in the complex frame $\xi_{1}, \ldots, \xi_{n}, \eta_{1}, \ldots, \eta_{n}$ defined by (35), $L_{\text {can }}$ and $g_{\text {can }}$ take the form:

$$
\begin{aligned}
& L_{\text {can }} \mapsto L_{\text {can }}^{\prime}=\left(\begin{array}{cccccccc}
\lambda & 1 & & & & & & \\
& \lambda & \ddots & & & & & \\
& & \ddots & 1 & & & & \\
& & & \lambda & & & & \\
& & & & \bar{\lambda} & 1 & & \\
& & & & \bar{\lambda} & \ddots & \\
& & & & & & \ddots & 1 \\
& & & & & & & \bar{\lambda}
\end{array}\right)=\left(\begin{array}{cc}
L_{\lambda} & 0 \\
0 & L_{\bar{\lambda}}
\end{array}\right),
\end{aligned}
$$

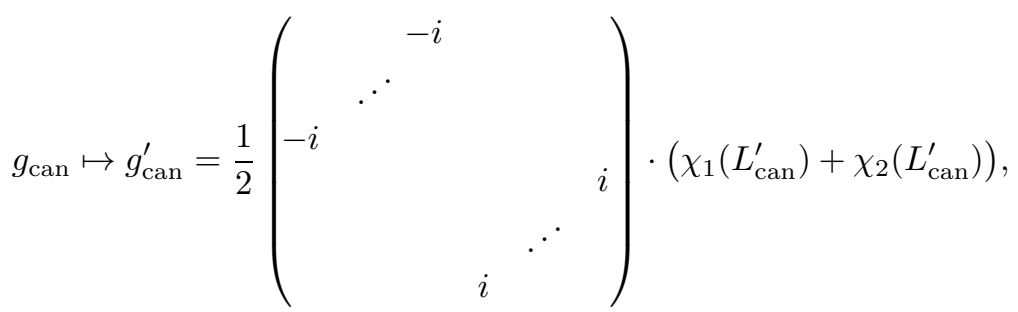

where $\chi_{1}(t)=(t-\lambda)^{n}$ and $\chi_{2}(t)=(t-\bar{\lambda})^{n}$ are the characteristic polynomials of $L_{\lambda}$ and $L_{\bar{\lambda}}$ respectively.

According to Lemma 3.3, we now can uniquely reconstruct the commutation relations between the elements of the frame $\xi_{1}, \ldots, \xi_{n}, \eta_{1}, \ldots, \eta_{n}$ and information about the derivatives of $\lambda$ and $\bar{\lambda}$ along these elements. This reconstruction could be done by straightforward computation. Instead it suffices to notice that we are now essentially in the same situation as in the case of two real Jordan blocks, but with $\lambda_{1}$ and $\lambda_{2}$ replaced by complex conjugate eigenvalues $\lambda$ and $\bar{\lambda}$; see formula (36) and the discussion around it. So we can repeat the above argument to get the conclusion of Proposition 4.1.

The canonical coordinate system $x_{1}, y_{1}, x_{2}, y_{2}, \ldots, x_{n}, y_{n}$ can now be reconstructed from these relations. To do it in the most natural way, we notice that $M$ carries a complex structure $J$ canonically associated with $L$. Indeed, we can introduce $J$ in an invariant way as follows. Let $L=L_{\mathrm{s}}+L_{\mathrm{n}}$ be the canonical 
decomposition of $L$ into semisimple and nilpotent parts. Then $J=\frac{1}{b}\left(L_{\mathrm{s}}-a\right)$ where $\lambda=a+i b$ is the eigenvalue of $L$. It is easy to see that $J^{2}=-\mathbf{1}$ and the integrability of $J$, i.e., vanishing of its Nijenhuis torsion $N_{J}$, follows from $N_{L}=0$.

Remark 4.2. The fact that the Nijenhuis torsion $N_{L}$ of $L$ vanishes is well known (see e.g. 6]); the implication $N_{L}=0 \Rightarrow N_{J}=0$ can be verified directly. Alternatively, one can use [9, Lemma 6]. Indeed, $J$ can be represented as the matrix function $J=f(L)$ that corresponds to the complex function $f: \mathbb{C} \backslash \mathbb{R} \rightarrow \mathbb{C}$, which is analytic, locally constant and defined in the following way: $f(a+i b)=i$ if $b>0$ and $f(a+i b)=-i$ if $b<0$. In a more general setting this construction of the canonical complex structure $J$ associated with $L$ is explained below in Lemma 5.2.

For the basis vectors $\xi_{k}$ and $\eta_{k}$ we have $J \xi_{k}=i \xi_{k}$ and $J \eta_{k}=-i \eta_{k}$. This means that for any holomorphic coordinate system $z_{1}, \ldots, z_{n}$, the vectors $\xi_{k}$ are linear combinations of $\partial_{z_{k}}$ and the $\eta_{k}$ 's are linear combinations of $\partial_{\bar{z}_{k}}$. Moreover, item 1 of Proposition 4.1 says that the vector fields $\xi_{1}, \ldots, \xi_{n}$ are holomorphic.

From now on we can forget about the $\eta_{k}$ 's and work with the $\xi_{k}$ 's only. The following repeats the arguments for a real Jordan block but in the complex (holomorphic) setting. Since the holomorphic vector fields $\xi_{1}, \ldots, \xi_{n-1}$ pairwise commute, we can find a local complex coordinate system $z_{1}, \ldots, z_{n}$ such that

$$
\xi_{k}=\partial_{z_{k}}, \quad k=1, \ldots, n-1 .
$$

Moreover, this coordinate system can be chosen in such a way that on the 2dimensional surface $z_{1}=z_{2}=\cdots=z_{n-1}=0$, we have

$$
\xi_{n}=\partial_{z_{n}} \text {. }
$$

The eigenvalue $\lambda$ is a holomorphic function (since $\xi_{k}$ 's are holomorphic and $\left.\left[\xi_{n-1}, \xi_{n}\right]=-(n-1) \xi_{n}(\lambda) \cdot \xi_{n}\right)$. Moreover, $\lambda$ depends on $z_{n}$ only because $\xi_{k}(\lambda)=$ $\partial_{z_{k}}(\lambda)=0, k=1, \ldots, n-1$.

Now our goal is to determine the transition matrix between two bases $\xi_{1}, \ldots, \xi_{n}$ and $\partial_{z_{1}}, \ldots, \partial_{z_{n}}$. This part of the proof repeats the arguments in the real case (see page 6737) and we omit it, referring to [10] for details. The final conclusion is that

$$
\partial_{z_{k}}=\xi_{k}, \quad k=1, \ldots, n-1, \quad \text { and } \quad \partial_{z_{n}}=\sum_{k=1}^{n} a_{k-1} \xi_{k},
$$

where $a_{i}$ are defined by the same formulas as (31) and (32) but with $x_{k}$ replaced by $z_{k}$.

Now we have all the information to rewrite the formulas for $L$ and $g$ in the basis $\partial_{z_{i}}$. Notice that $L$ commutes with the complex structure $J$ and therefore $L$ can be treated as a complex operator. The form $g$ is also compatible with $J$ in the sense that $g(J u, v)=g(u, J v)$ so that $g$ can be understood as the real part of the complex bilinear form on $T_{p} M$ treated as an $n$-dimensional complex space (w.r.t. $J$ ). This allows us to represent $L$ and $g$ by $n \times n$ complex matrices. In the canonical frame $\xi_{1}, \ldots, \xi_{n}$ these matrices are

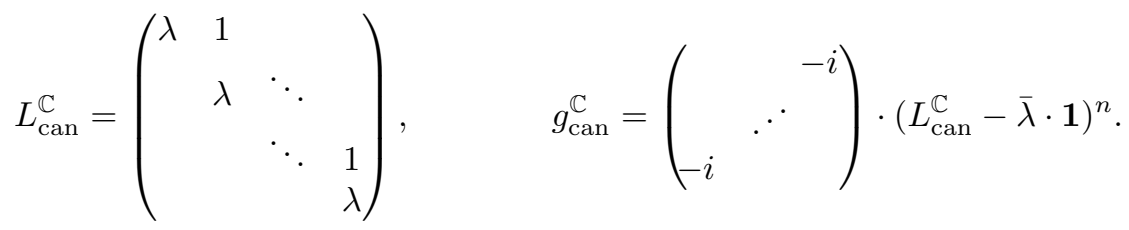


To determine $L$ and $g$ (more precisely their complex representations $L^{\mathbb{C}}$ and $g^{\mathbb{C}}$ ) in the coordinates $z_{1}, \ldots, z_{n}$, we use the standard transformation

$$
L_{\text {can }}^{\mathbb{C}} \mapsto L^{\mathbb{C}}=C^{-1} L_{\text {can }}^{\mathbb{C}} C, \quad g_{\text {can }}^{\mathbb{C}} \mapsto g^{\mathbb{C}}=C^{\top} g_{\text {can }}^{\mathbb{C}} C
$$

with the transition matrix $C$ :

$$
\left(\partial_{z_{1}}, \ldots, \partial_{z_{n}}\right)=\left(\xi_{1}, \ldots, \xi_{n}\right) C, \quad C=\left(\begin{array}{ccccc}
1 & & & & a_{0} \\
& 1 & & & a_{1} \\
& & \ddots & & \vdots \\
& & & 1 & a_{n-2} \\
& & & & a_{n-1}
\end{array}\right) .
$$

Now a straightforward computation of $g^{\mathbb{C}}$ and $L^{\mathbb{C}}$ immediately leads to the conclusion of Theorem 1.9 page 6728

\section{Applications: Some global Results}

Here we give the proofs of Theorem 1.11 and Corollary 1.12 page 6730 .

5.1. Proof of Theorem 1.11. Consider two projectively equivalent pseudoRiemannian metrics $g$ and $\bar{g}$ on $M$ and the $(1,1)$-tensor fields $L=L(g, \bar{g})$ defined by (3) which we repeat for the convenience of the reader here:

$$
L_{j}^{i}=\left|\frac{\operatorname{det} \bar{g}}{\operatorname{det} g}\right|^{\frac{1}{n+1}} \bar{g}^{i k} g_{k j} .
$$

Theorem 1.11 can be reformulated as follows:

If $M$ is compact, then nonreal eigenvalues of $L$ are all constant.

The idea of the proof is very natural. As we know from 4 , a complex nonreal eigenvalue of $L$ is a holomorphic function in an appropriate coordinate system. Roughly speaking, our proof is somehow equivalent to saying that "a holomorphic function on a compact manifold has to be constant." However, to make sense out of this principle we have to deal with two issues:

- the complex structure $J$ (see Theorem 1.9 and §4) is not globally defined;

- the eigenvalues of $L$ may collide and near the collision points they cannot be considered as well-defined functions (these points should be treated as branching points for eigenvalues).

To avoid these difficulties, we use the following two observations:

- a natural complex structure $J$ is well defined as soon as we have complex nonreal eigenvalues even at collision points,

- the complex nonreal eigenvalues $\lambda_{i}$ of $L$ can be replaced by symmetric polynomials of them, like $\sum \lambda_{i}$, which are still holomorphic and well defined even at collision points.

These two ideas are formalized in the following lemma. Let $\chi_{L}(t)$ be the characteristic polynomial of $L$. Clearly, the coefficients of $\chi_{L}(t)$ are smooth real functions on $M$.

Lemma 5.1. Let $\mu_{0}$ be a complex root of $\chi_{L}(t)$ of multiplicity $k$ at a point $p_{0} \in$ $M, \operatorname{Im} \mu_{0}>0$. Then in a neighborhood of $p_{0}$ there is a local coordinate system 
$x_{1}, \ldots, x_{k}, y_{1}, \ldots, y_{k}, v_{1}, \ldots, v_{l}, 2 k+l=\operatorname{dim} M$ such that the characteristic polynomial of $L$ admits the following factorization:

$$
\chi_{L}(t)=P_{z}(t) \cdot \bar{P}_{z}(t) \cdot Q_{v}(t) k,
$$

where

$$
P_{z}(t)=t^{k}+a_{k-1}(z) t^{k-1}+\cdots+a_{1}(z) t+a_{0}(z)
$$

with coefficients $a_{m}(z)$ being holomorphic functions of the complex variables $z_{j}=$ $x_{j}+i y_{j}$,

and

$$
\bar{P}_{z}(t)=t^{k}+\bar{a}_{k-1}(z) t^{k-1}+\cdots+\bar{a}_{1}(z) t+\bar{a}_{0}(z)
$$

$$
Q_{v}(t)=t^{l}+b_{l-1}(v) t^{l-1}+\cdots+b_{1}(v) t+b_{0}(v)
$$

where $b_{m}(v)$ are smooth real valued functions of $v^{1}, \ldots, v^{l}$, and the polynomial $P_{z}(t)$ at the point $p_{0}$ takes the form $\left(t-\mu_{0}\right)^{k}$.

Proof. We first notice (as we did in our splitting construction [9]) that in a neighborhood of $p_{0}$ the characteristic polynomial $\chi_{L}(t)$ can be uniquely factorized into two monic polynomials of degree $2 k$ and $l$ respectively with smooth real coefficients

$$
\chi_{L}(t)=\chi_{1}(t) \chi_{2}(t)
$$

in such a way that the roots of $\chi_{1}(t)$ at the point $p_{0}$ are $\mu_{0}$ and $\bar{\mu}_{0}$, both with multiplicity $k$. Locally, in a neighborhood of $p_{0}$ these polynomials do not have common roots. This factorization immediately leads (see [9, Theorem 2]) to the existence of a coordinate system $u^{1}, \ldots, u^{2 k}, v^{1}, \ldots, v^{l}$ in which $L$ splits into blocks each of which depends on its own group of coordinates:

$$
L=\left(\begin{array}{cc}
L_{1}(u) & 0 \\
0 & L_{2}(v)
\end{array}\right)
$$

so that $\chi_{1}(t)$ and $\chi_{2}(t)$ are the characteristic polynomials of $L_{1}$ and $L_{2}$. In other words, locally we may think of $M$ with $L$ as a direct product $\left(M_{1}, L_{1}\right) \times\left(M_{2}, L_{2}\right)$ of two "independent" manifolds with $(1,1)$-tensor fields on them. We put $Q_{v}(t)=$ $\chi_{2}(t)$ and continue working, from now on, with the first factor $\left(M_{1}, L_{1}\right)$ only.

In a neighborhood of $p_{0}$, the characteristic polynomial $\chi_{1}(t)$ of $L_{1}$ admits a further factorization:

$$
\chi_{1}(t)=P_{u}(t) \bar{P}_{u}(t)
$$

into two complex conjugate polynomials with smooth complex valued coefficients satisfying the required property: at the point $p_{0}$ we have $P_{u}(t)=\left(t-\mu_{0}\right)^{k}$. So far this construction is purely algebraic. But now we need to pass from real coordinates $u$ to complex coordinates $z=x+i y$ in such a way that the coefficients of $P_{u}(t)$ become holomorphic functions of $z$.

First we construct a complex structure $J$ on $M_{1}$ canonically associated with $L_{1}$. Since this construction seems to be quite natural and perhaps useful in other geometric problems, we explain it in a more general context, referring to $[9], \S 1.3$ for technical details.

Consider a locally constant complex function $f: \mathbb{C} \backslash \mathbb{R} \rightarrow \mathbb{C}$ defined by

$$
f(z)=\left\{\begin{aligned}
i & \text { if } \operatorname{Im} z>0 \\
-i & \text { if } \operatorname{Im} z<0
\end{aligned}\right.
$$

Since $f$ can be uniformly approximated (with all derivatives up to any fixed order) by polynomials with real coefficients on every compact subset $K \subset \mathbb{C} \backslash \mathbb{R}$, we are also 
allowed to consider $f$ as a real analytic matrix function $f: U \rightarrow \operatorname{End}\left(\mathbb{R}^{n}\right)$, where $U \subset \operatorname{End}\left(\mathbb{R}^{n}\right)$ is a subset of all operators with no real eigenvalues (in particular, here $n=2 k$ ).

Now, let $L$ be a smooth $(1,1)$-tensor field on a smooth manifold $M$ with no real eigenvalues (like $L_{1}$ on $M_{1}$ in our case); i.e., the spectrum of $L$ at every point $x \in M$ belongs to $\mathbb{C} \backslash \mathbb{R}$. Then we can build a new $(1,1)$-tensor field $J$ on $M$ by setting $J=f(L)$ pointwise.

Lemma 5.2. The $(1,1)$-tensor field $J$ satisfies the following properties:

(1) $J$ is smooth.

(2) $J^{2}=-\mathbf{1}$; i.e. $J$ is an almost complex structure on $M$.

(3) $J L=L J$; i.e. $L$ is a complex linear operator w.r.t. $J$.

(4) If the Nijenhuis torsion $N_{L}$ of $L$ vanishes, then $J$ is integrable and hence is a complex structure on $M$.

Proof. The smoothness of $J$ follows from the fact that $f: U \rightarrow \operatorname{End}\left(\mathbb{R}^{n}\right)$ is a real analytic matrix function and $L$ depends smoothly of $x \in M$. Next, items (2) and (3) are purely algebraic. Indeed, the scalar identity $f^{2}(z) \equiv-1$ implies the matrix identity $J^{2}=f(L) f(L)=-\mathbf{1}$, and $L$ commutes with $f(L)$ for any matrix function $f$. Finally, the integrability of $J$, i.e., the fact that $N_{J} \equiv 0$, is a particular case of Lemma 6 from 9 .

Remark 5.3. An equivalent definition of $J$, as a function of $L$, is as follows. Let $L: V^{2 k} \rightarrow V^{2 k}$ be a real linear operator with no real eigenvalues. We consider the decomposition of $V^{\mathbb{C}}$ into two $L$-invariant subspaces $V^{+} \oplus V^{-}$corresponding to the eigenvalues of $L$ with positive and negative imaginary parts respectively. Such a decomposition is obviously unique. Now we define $J$ to be the multiplication by $i$ on $V^{+}$and multiplication by $-i$ on $V^{-}$. It is easy to see that $V$ as a subspace of $V^{\mathbb{C}}$ is $J$-invariant; i.e., $J$ gives a well-defined operator on $V$, satisfying $J^{2}=-\mathbf{1}$ and commuting with $L$. In particular, in the case of a single complex Jordan block, the complex structure $J$ canonically associated with $L$ coincides with the one we used in 4 (cf. Remark 4.2. page 6741). Moreover, in a neighborhood of a regular point, $J$ is the direct sum of the complex structures constructed for each individual $\left(\lambda_{i}, \bar{\lambda}_{i}\right)$-block by the method explained in 4

Let us come back to the proof of Lemma 5.1. By applying Lemma 5.2 to the $(1,1)$-tensor field $L_{1}$ on $M_{1}$, we construct the complex structure $J$ on $M_{1}$ canonically associated with $L_{1}$. Next, we need to show that the coefficients of $P_{z}(t)$ (obtained from $P_{u}(t)$ by replacing the coordinates $u$ with $z$ ) are holomorphic with respect to $J$. Though it can be done independently, we shall easily derive this property from 4

Indeed, in 4 we have shown that in a neighborhood of every regular point each complex nonreal eigenvalue $\lambda_{i}$ of $L$ is a holomorphic function (in the "singular" case studied in $2, \lambda_{i}$ is constant, so this property holds automatically) w.r.t. the complex structure associated with the $\left(\lambda_{i}, \bar{\lambda}_{i}\right)$-block. Taking into account Remark 5.3. we see that each $\lambda_{i}$ is holomorphic w.r.t. $J$. On the other hand, the coefficients $a_{j}(z)$ of $P_{z}(t)$ are symmetric polynomials in $\lambda_{i}$, so they are holomorphic at each regular point too. Now it remains to notice that regular points form an open dense subset and $a_{j}(z)$ are smooth everywhere. This obviously implies that $a_{j}(z)$ are holomorphic on the whole neighborhood of $p_{0}$. This completes the proof. 
We shall also use the following almost obvious statement.

Lemma 5.4. Let $P_{z}(t)=t^{k}+a_{k-1}(z) t^{k-1}+\cdots+a_{1}(z) t+a_{0}(z)$ be a polynomial in $t$ whose coefficients are holomorphic functions of $z=\left(z_{1}, \ldots, z_{k}\right) \in U$, where $U \subset \mathbb{C}^{k}$ is an open connected domain.

Assume that at some point $z_{0} \in U$ the polynomial takes the form $P_{z_{0}}(t)=$ $\left(t-\mu_{0}\right)^{k}$ and at any other point $z \in U$ all the roots $\lambda_{i}(z)$ of $P_{z}(t)$ satisfy the condition

$$
\operatorname{Im} \lambda_{i}(z) \leq c=\operatorname{Im} \mu_{0}, \quad i=1, \ldots, k .
$$

Then $\lambda_{i}(z) \equiv \mu_{0}$ for all $z \in U$; i.e., the roots of $P_{z}(t)$ are all constant and equal to $\mu_{0}$. In particular, $P_{z}(t) \equiv\left(t-\mu_{0}\right)^{k}$ on $U$.

Proof. Consider the sum $\sum_{i=1}^{k} \lambda_{i}(z)$ of the roots of $P_{z}(t)$. Since $\sum_{i=1}^{k} \lambda_{i}(z)=$ $-a_{k-1}(z)$, this sum is a holomorphic function on $U$. On the other hand, we see that $\operatorname{Im}\left(-a_{k-1}(z)\right)=\operatorname{Im}\left(\sum_{i=1}^{k} \lambda_{i}(z)\right) \leq k \cdot c$ and $\operatorname{Im}\left(-a_{k-1}\left(z_{0}\right)\right)=k \cdot c$; i.e., the imaginary part of the holomorphic function $-a_{k-1}(z)$ attains a maximum at a certain point $z_{0} \in U$. This implies (by the maximum principle) that $a_{k-1}(z)$ is constant on $U$. From this, in turn, it is easy to derive that the imaginary part of each $\lambda_{i}(z)$ and, therefore, $\lambda_{i}(z)$ itself is constant.

We are now ready to complete the proof of Theorem 1.11

Consider the roots $\lambda_{1}(p), \ldots, \lambda_{n}(p), n=\operatorname{dim} M$ of the characteristic polynomial $\chi_{L}(t)$ at $p \in M$ and let

$$
c=\max _{p \in M, i=1, \ldots, n} \operatorname{Im} \lambda_{i}(p) .
$$

We assume that some complex eigenvalues exist, so $c>0$.

Since the roots $\lambda_{i}(p)$ depend on $p$ continuously (in a natural sense) and $M$ is compact, then $c$ is attained; i.e., there is a point at which $\chi_{L}(t)$ has a complex root $\mu_{0}$ such that $\operatorname{Im} \mu_{0}=c$. In general, $\mu_{0}$ may have different multiplicities at different points. Let $k$ be the maximal multiplicity of $\mu_{0}$ on $M$.

Consider the following subset $A \subset M$ :

$$
A=\left\{q \in M \mid \mu_{0} \text { is a root of } \chi_{L}(t) \text { of multiplicity } k \text { at the point } q\right\} .
$$

By our assumption, $A$ is nonempty and, as the multiplicity of $\mu_{0}$ is upper semicontinuous and $k$ is its maximum, $A$ is closed. Let us show that $A$ is open. Indeed, let $p_{0} \in A$. We first apply Lemma 5.1 at this point to get the factorization (38) in some neighborhood $U\left(p_{0}\right)$ and then apply Lemma 5.4 to see that $\chi_{L}(t)=$ $\left(t-\mu_{0}\right)^{k}\left(t-\bar{\mu}_{0}\right)^{k} Q_{v}(t)$ on $U\left(p_{0}\right)$. In other words, $\mu_{0}$ is a root of $\chi_{L}(t)$ of multiplicity $k$ for all points $p \in U\left(p_{0}\right)$, i.e. $U\left(p_{0}\right) \subset A$ and therefore $A$ is open.

Thus, $A$ is open, closed and nonempty. Hence, $A=M$, and we see that $\chi_{L}(t)=$ $\left(t-\mu_{0}\right)^{k}\left(t-\bar{\mu}_{0}\right)^{k} Q_{v}(t)$ everywhere on $M$. In other words, $\mu_{0}$ is a constant complex eigenvalue of $L$ of multiplicity $k$ on the whole manifold $M$.

If $Q_{v}(t)$ has some other nonreal roots at some points of $M$, we simply repeat the same argument to show that these roots have to be constant. This completes the proof of the first statement of Theorem 1.11 .

In order to proof that the multiplicity of a nonreal eigenvalue is the same at every point, it is sufficient to observe that otherwise there must be a nonreal nonconstant eigenvalue of $L$, which contradicts the proven part of Theorem 1.11 . 
5.2. Proof of Corollary 1.12, We need to prove the following result:

Let $M^{3}$ be a closed connected 3-dimensional manifold. Suppose $g$ and $\bar{g}$ are geodesically equivalent metrics on it and L, given by (21), has a nonreal eigenvalue at least at one point. Then, $M^{3}$ can be finitely covered by the 3-torus.

Without loss of generality we assume that the metric $g$ has signature $(-,+,+)$. Then, by Theorem 1.11, page 6730, a nonreal eigenvalue of $L$ is a constant; we denote it by $\alpha+i \beta$. The complex conjugate number $\alpha-i \beta$ is also an eigenvalue of $L$; the remaining third eigenvalue will be denoted by $\lambda$; it is a (smooth) real valued function on the manifold.

At every point $p \in M$, let us consider a basis $\left\{v_{1}, v_{2}, v_{3}\right\}$ in $T_{p} M$ such that in this basis the matrices of $g$ and $L$ are given by

$$
g=\left(\begin{array}{ccc}
(\lambda-\alpha)^{2}+\beta^{2} & & \\
& -\beta & \alpha-\lambda \\
& \alpha-\lambda & \beta
\end{array}\right), \quad L=\left(\begin{array}{ccc}
\lambda & & \\
& \alpha & \beta \\
-\beta & \alpha
\end{array}\right) .
$$

The existence of such a basis follows from [28, Theorem 12.2]; it is an easy exercise to show that the basis is unique up to the transformations $v_{1} \mapsto-v_{1} ;\left(v_{2}, v_{3}\right) \mapsto$ $\left(-v_{2},-v_{3}\right)$.

Now, consider the positive definite Euclidean structure at $T_{p} M$ such that this basis is orthonormal. This Euclidean structure does not depend on the freedom in the choice of the basis and is therefore well defined. It smoothly depends on the point $p$ and therefore generates a Riemannian metric on $M$, which we denote by $g_{0}$. Let us show that the metric $g_{0}$ is flat.

In order to do this, we will use our description of compatible pairs $(g, L)$. As we explained in $\$ 1.2$, in a neighborhood of every point the metric $g$ could be obtained by gluing $\left(I, h_{1}, L_{1}\right)$ and $\left(U^{2}, h_{2}, L_{2}\right)$ where

- $I$ is 1 -dimensional, the metric $h_{1}$ is positive definite and the eigenvalue of $L_{1}$ is $\lambda$.

- $U^{2}$ is 2-dimensional, $h_{2}$ has signature $(-,+)$, the eigenvalues of $L_{2}$ are $\alpha+i \beta, \alpha-i \beta$ and $h_{2}$ and $L_{2}$ are compatible.

Then, for a certain choice of the coordinate $x_{1}$ on $I$ the metric $h_{1}$ is $\left(d x_{1}\right)^{2}$ and the only component of the $(1 \times 1)$-matrix of $L$ is $\lambda\left(x_{1}\right)$. Now, since $L_{2}$ is compatible with $h_{2}$ and since the trace of $L_{2}$ is constant, we conclude from (5) that $L_{2}$ is covariantly constant with respect to $h_{2}$. Then, $h_{2}$ is obviously flat, and in a certain (local) coordinate system $\left(x_{2}, x_{3}\right)$ on $U^{2}$ the metric $h_{2}$ and the $(1,1)$-tensor $L$ are given by the matrices

$$
h_{2}=\left(\begin{array}{ll}
0 & 1 \\
1 & 0
\end{array}\right), \quad L_{2}=\left(\begin{array}{cc}
\alpha & \beta \\
-\beta & \alpha
\end{array}\right)
$$

Applying the gluing construction to $\left(I, h_{1}, L_{1}\right)$ and $\left(U^{2}, h_{2}, L_{2}\right)$, we obtain that the metric $g$ and the tensor $L$ are given by

$$
g=\left(\begin{array}{ccc}
\left(\lambda\left(x_{1}\right)-\alpha\right)^{2}+\beta^{2} & & \\
& -\beta & \alpha-\lambda\left(x_{1}\right) \\
& \alpha-\lambda\left(x_{1}\right) & \beta
\end{array}\right), \quad L=\left(\begin{array}{ccc}
\lambda\left(x_{1}\right) & & \\
& \alpha & \beta \\
& -\beta & \alpha
\end{array}\right) .
$$

We see that the vector fields $v_{1}=\frac{\partial}{\partial x_{1}}, v_{2}=\frac{\partial}{\partial x_{2}}, v_{3}=\frac{\partial}{\partial x_{3}}$ form a basic such that $g$ and $L$ are as in (39), implying that the metric $g_{0}$ is given by $g_{0}=\left(d x_{1}\right)^{2}+\left(d x_{2}\right)^{2}+$ $\left(d x_{3}\right)^{2}$ and therefore is flat. 
Thus, there exists a flat Riemannian metric on $M^{3}$. Then, the manifold is a 3-dimensional Bieberbach manifold (i.e., is a quotient of $\mathbb{R}^{3}$ modulo a freely acting crystallographic group) and can be finitely covered by the torus $T^{3}$ as we claimed.

\section{ACKNowledgements}

The authors are very grateful to the anonymous referee for numerous comments and suggestions. They also thank David Dowell and Eugenie Hunsicker for grammatical and stylistic corrections.

\section{REFERENCES}

[1] A. V. Aminova, Pseudo-Riemannian manifolds with general geodesics (Russian), Uspekhi Mat. Nauk 48 (1993), no. 2(290), 107-164, DOI 10.1070/RM1993v048n02ABEH001014; English transl., Russian Math. Surveys 48 (1993), no. 2, 105-160. MR.1239862 (94h:53088)

[2] A. V. Aminova, Projective transformations of pseudo-Riemannian manifolds, J. Math. Sci. (N. Y.) 113 (2003), no. 3, 367-470, DOI 10.1023/A:1021041802041. Geometry, 9. MR1965077 (2004a:53089)

[3] E. Beltrami, Risoluzione del problema: riportare $i$ punti di una superficie sopra un piano in modo che le linee geodetiche vengano rappresentate da linee rette, Ann. Mat. 1 (1865), no. 7, 185-204.

[4] E. Beltrami, Saggio di interpetrazione della geometria non-euclidea, Giornale di matematiche, vol. VI (1868).

[5] E. Beltrami, Teoria fondamentale degli spazii di curvatura costante, Annali. di Mat., ser. II 2 (1968), 232-255.

[6] Alexey V. Bolsinov and Vladimir S. Matveev, Geometrical interpretation of Benenti systems, J. Geom. Phys. 44 (2003), no. 4, 489-506, DOI 10.1016/S0393-0440(02)00054-2. MR1943174 (2005d:37117)

[7] Alexey V. Bolsinov, Vladimir S. Matveev, and Giuseppe Pucacco, Normal forms for pseudoRiemannian 2-dimensional metrics whose geodesic flows admit integrals quadratic in momenta, J. Geom. Phys. 59 (2009), no. 7, 1048-1062, DOI 10.1016/j.geomphys.2009.04.010. MR2536861 (2010f:53153)

[8] Alexey V. Bolsinov, Vladimir S. Matveev, G. Pucacco, Dini theorem for pseudo-Riemannian metrics, Math. Ann. 352 (2012), 900-909, arXiv:math/0802.2344.

[9] Alexey V. Bolsinov and Vladimir S. Matveev, Splitting and gluing lemmas for geodesically equivalent pseudo-Riemannian metrics, Trans. Amer. Math. Soc. 363 (2011), no. 8, 40814107, DOI 10.1090/S0002-9947-2011-05187-1. MR2792981(2012f:53145)

[10] Alexey V. Bolsinov and Vladimir S. Matveev, Local normal forms for geodesically equivalent pseudo-Riemannian metrics, arXiv:1301.2492.

[11] Alexey V. Bolsinov and Dragomir Tsonev, On a new class of holonomy groups in pseudoRiemannian geometry, J. Differential Geom. 97 (2014), no. 3, 377-394. MR3233515

[12] Ch. Boubel, On the algebra of parallel endomorphisms of a pseudo-Riemannian metric, to appear in J. Diff. Geom. arXiv:math.DG/1207.6544.

[13] Robert L. Bryant, Gianni Manno, and Vladimir S. Matveev, A solution of a problem of Sophus Lie: normal forms of two-dimensional metrics admitting two projective vector fields, Math. Ann. 340 (2008), no. 2, 437-463, DOI 10.1007/s00208-007-0158-3. MR2368987 (2008m:53037)

[14] Robert Bryant, Maciej Dunajski, and Michael Eastwood, Metrisability of two-dimensional projective structures, J. Differential Geom. 83 (2009), no. 3, 465-499. MR.2581355 (2011f:53043)

[15] Lennart Carleson, Mergelyan's theorem on uniform polynomial approximation, Math. Scand. 15 (1964), 167-175. MR0198209 (33 \#6368) 
[16] Gaston Darboux, Leçons sur la théorie générale des surfaces. III, IV (French), Les Grands Classiques Gauthier-Villars. [Gauthier-Villars Great Classics], Éditions Jacques Gabay, Sceaux, 1993. Lignes géodésiques et courbure géodésique. Paramètres différentiels. Déformation des surfaces. [Geodesic lines and geodesic curvature. Differential parameters. Deformation of surfaces]; Déformation infiniment petite et représentation sphérique. [Infinitely small deformation and spherical representation]; Reprint of the 1894 original (III) and the 1896 original (IV); Cours de Géométrie de la Faculté des Sciences. [Course on Geometry of the Faculty of Science]. MR1365962 (97c:01046b)

[17] U. Dini, Sopra un problema che si presenta nella teoria generale delle rappresentazioni geografice di una superficie su un'altra, Ann. di Math., ser. 2, 3 (1869), 269-293.

[18] Michael Eastwood and Vladimir Matveev, Metric connections in projective differential geometry, Symmetries and overdetermined systems of partial differential equations, IMA Vol. Math. Appl., vol. 144, Springer, New York, 2008, pp. 339-350, DOI 10.1007/978-0-387-738314_16. MR2384718 (2009a:53018)

[19] Luther Pfahler Eisenhart, Non-Riemannian geometry, American Mathematical Society Colloquium Publications, vol. 8, American Mathematical Society, Providence, RI, 1990. Reprint of the 1927 original. MR 1466961 (98j:53001)

[20] Luther Pfahler Eisenhart, The geometry of paths and general relativity, Ann. of Math. (2) 24 (1923), no. 4, 367-392, DOI 10.2307/1967681. MR1502647

[21] G. Fubini, Sui gruppi transformazioni geodetiche, Mem. Acc. Torino 53 (1903), 261-313.

[22] V. I. Golikov, Geodesic mapping of gravitational fields of general type (Russian), Trudy Sem. Vektor. Tenzor. Anal. 12 (1963), 97-129. MR0159670 (28 \#2887)

[23] Nicholas J. Higham, Functions of matrices, Theory and computation, Society for Industrial and Applied Mathematics (SIAM), Philadelphia, PA, 2008. MR2396439 (2009b:15001)

[24] Volodymyr Kiosak and Vladimir S. Matveev, Complete Einstein metrics are geodesically rigid, Comm. Math. Phys. 289 (2009), no. 1, 383-400, DOI 10.1007/s00220-008-0719-7. MR2504854 (2011b:53104)

[25] G. I. Kručkovič and A. S. Solodovnikov, Constant symmetric tensors in Riemannian spaces (Russian), Izv. Vysš. Učebn. Zaved. Matematika 1959 (1959), no. 3 (10), 147-158. MR0133084 (24 \#A2920)

[26] G. I. Kručkovič, Equations of semireducibility and geodesic correspondence of Lorentz spaces, Trudy Vsecsoyuz. Zaochn. Energet. Inst. 24 (1963), 74-87.

[27] J.-L. Lagrange, Sur la construction des cartes géographiques, Novéaux Mémoires de l'Académie des Sciences et Bell-Lettres de Berlin, 1779.

[28] Peter Lancaster and Leiba Rodman, Canonical forms for Hermitian matrix pairs under strict equivalence and congruence, SIAM Rev. 47 (2005), no. 3, 407-443 (electronic), DOI 10.1137/S003614450444556X. MR2178635(2007i:15021)

[29] David B. Leep and Laura Mann Schueller, Classification of pairs of symmetric and alternating bilinear forms, Exposition. Math. 17 (1999), no. 5, 385-414. MR1733879 (2000m:11033)

[30] T. Levi-Civita, Sulle trasformazioni delle equazioni dinamiche, Ann. di Mat., serie $2^{a}, 24$ (1896), 255-300.

[31] Sophus Lie, Untersuchungen über geodätische Curven (German), Math. Ann. 20 (1882), no. 3, 357-454, DOI 10.1007/BF01443601. MR1510173

[32] R. Liouville, Sur les invariants de certaines équations différentielles et sur leurs applications, Journal de l'École Polytechnique 59 (1889), 7-76.

[33] Vladimir S. Matveev and Peter $\breve{\mathrm{I}}$. Topalov, Trajectory equivalence and corresponding integrals (English, with English and Russian summaries), Regul. Chaotic Dyn. 3 (1998), no. 2, 30-45, DOI 10.1070/rd1998v003n02ABEH000069. MR.1693470(2000d:37068)

[34] Vladimir S. Matveev, Hyperbolic manifolds are geodesically rigid, Invent. Math. 151 (2003), no. 3, 579-609, DOI 10.1007/s00222-002-0263-6. MR1961339 (2004f:53044)

[35] Vladimir S. Matveev, On projectively equivalent metrics near points of bifurcation, Topological methods in the theory of integrable systems, Camb. Sci. Publ., Cambridge, 2006, pp. 215-240. MR2454556(2010c:53124)

[36] Vladimir S. Matveev, Proof of the projective Lichnerowicz-Obata conjecture, J. Differential Geom. 75 (2007), no. 3, 459-502. MR2301453 (2007m:53030)

[37] Vladimir S. Matveev, Geodesically equivalent metrics in general relativity, J. Geom. Phys. 62 (2012), no. 3, 675-691, DOI 10.1016/j.geomphys.2011.04.019. MR2876790(2012m:53080) 
[38] Vladimir S. Matveev, Two-dimensional metrics admitting precisely one projective vector field, Math. Ann. 352 (2012), no. 4, 865-909, DOI 10.1007/s00208-011-0659-y. MR2892455

[39] J. Mikeš, Geodesic mappings of affine-connected and Riemannian spaces, J. Math. Sci. 78 (1996), no. 3, 311-333, DOI 10.1007/BF02365193. Geometry, 2. MR1384327 (97b:53043)

[40] P. Painlevé, Sur les intégrale quadratiques des équations de la Dynamique, Compt. Rend. 124 (1897), 221-224.

[41] A. Z. Petrov, Geodesic mappings of Riemannian spaces of an indefinite metric (Russian), Uchen. Zap. Kazan. Univ. 109 (1949), no. 3, 7-36.

[42] N. S. Sinjukov, Geodezicheskie otobrazheniya rimanovykh prostranstv (Russian), "Nauka", Moscow, 1979. MR552022 (81g:53014)

[43] A. S. Solodovnikov, Projective transformations of Riemannian spaces (Russian), Uspehi Mat. Nauk (N.S.) 11 (1956), no. 4(70), 45-116. MR.0084826 (18,930b)

[44] Peter Topalov, Geodesic compatibility and integrability of geodesic flows, J. Math. Phys. 44 (2003), no. 2, 913-929, DOI 10.1063/1.1526939. MR.1953103 (2003j:37088)

[45] T. Y. Thomas, On the projective theory of two dimensional Riemann spaces, Proc. Nat. Acad. Sci. U. S. A. 31 (1945), 259-261. MR0012520 (7,33g)

[46] Oswald Veblen and Tracy Yerkes Thomas, The geometry of paths, Trans. Amer. Math. Soc. 25 (1923), no. 4, 551-608, DOI 10.2307/1989307. MR 1501260

[47] Oswald Veblen and Joseph Miller Thomas, Projective invariants of affine geometry of paths, Ann. of Math. (2) 27 (1926), no. 3, 279-296, DOI 10.2307/1967848. MR1502733

[48] H. Weyl, Zur Infinitisimalgeometrie: Einordnung der projektiven und der konformen Auffasung, Nachrichten von der K. Gesellschaft der Wissenschaften zu Göttingen, MathematischPhysikalische Klasse, 1921; "Selecta Hermann Weyl”, Birkhäuser Verlag, Basel und Stuttgart, 1956.

School of Mathematics, Loughborough University, Loughborough, LE11 3TU, United KINGDOM

E-mail address: A.Bolsinov@lboro.ac.uk

Institute of Mathematics, Friedrich-Schiller University Jena, 07737, Jena, Germany

E-mail address: vladimir.s.matveev@gmail.com 